\title{
Economia compartilhada e governança pública
}

\section{Adilson Giovanini 1}

1 Universidade do Estado de Santa Catarina / Departamento de Governança Pública, Balneário Camboriú / SC - Brasil

O termo economia compartilhada é empregado na literatura especializada para identificar a forma como a internet, os smartphones e os aplicativos estão modificando a dinâmica econômica mundial. Este artigo apresenta uma pesquisa documental realizada para identificar os aplicativos de compartilhamento que surgiram nas últimas décadas e o modo como estão contribuindo para a melhoria da gestão pública local. Utilizou-se a análise descritiva dos dados e uma regressão para caracterizar a adesão às novas tecnologias pelos governos locais e para identificar o modo como afetam o desempenho fiscal dos municípios, mensurado a partir do Índice FIRJAN de Gestão Fiscal (IFGF). Os resultados obtidos mostram que os aplicativos de economia compartilhada podem contribuir de diferentes formas, com destaque para: a maior cooperação e coordenação intra e entre governos locais, redução na subutilização de ativos, maior acesso e melhoria na qualidade dos serviços públicos, e maior interação e participação dos cidadãos nas decisões públicas. A regressão estimada mostra que o emprego das novas tecnologias de comunicação contribui para a melhoria no desempenho fiscal dos municípios. Contudo, essas tecnologias são pouco utilizadas, sendo necessárias iniciativas que estimulem a utilização de aplicativos de compartilhamento nas gestões públicas locais. Palavras-chave: economia compartilhada; municípios; gestão pública local.

\section{Economía compartida y gobernanza pública}

El término economía compartida se utiliza en la literatura especializada para identificar cómo la Internet, los smartphones y las aplicaciones están cambiando la dinámica económica mundial. Este artículo presenta una investigación documental realizada para identificar las aplicaciones de compartición que surgieron en las últimas décadas y cómo están contribuyendo a la mejora de la gestión pública local. Se utilizó el análisis descriptivo de datos y la regresión para caracterizar la adhesión a las nuevas tecnologías por parte de los gobiernos locales y para identificar cómo afectan el desempeño fiscal de los municipios, medido a partir del Índice FIRJAN de Gestión Fiscal (IFGF). Los resultados obtenidos muestran que las aplicaciones de economía compartida pueden contribuir de diferentes formas, con énfasis en: mayor cooperación y coordinación dentro y entre los gobiernos locales, reducción de la subutilización de activos, mayor acceso y mejora en la calidad de los servicios públicos y mayor interacción y participación ciudadana en las decisiones públicas. La regresión estimada muestra que el uso de nuevas tecnologías de la comunicación contribuye a mejorar el desempeño fiscal de los municipios. Sin embargo, estas tecnologías rara vez se utilizan y se necesitan iniciativas para fomentar el uso de aplicaciones de compartición en las gestiones públicas locales.

Palabras clave: economía compartida; municipios; gestión pública local.

\section{Sharing economy and public governance}

The term sharing economy is used in specialized literature to identify how the Internet, smartphones, and applications are changing the global economic dynamic. This article presents documentary research focused on private sharing applications that have emerged in recent decades, intending to contribute to the improvement of local public management. Descriptive data analysis and regression were used to characterize the local governments' adherence to new technologies and to identify how these new technologies affect the fiscal performance of municipalities measured by the FIRJAN Fiscal Management Index. The results obtained show that shared economy Apps can contribute in different ways, with emphasis on greater cooperation and coordination within and between local governments, reduction in the underutilization of assets, greater access and improvement in the quality of public services, and greater interaction and citizen participation in public decisions. The estimated regression shows that the use of new communication technologies contributes to improving the municipalities' fiscal performance. However, these technologies are little used and should be encouraged in local public administrations.

Keywords: shared economy; counties; local public management. 


\section{INTRODUÇÃO}

O termo "economia compartilhada" (sharing economy ou shared economy) surgiu nos Estados Unidos na década de 1930, no contexto da Grande Depressão, e está relacionado à emergência de tecnologias sociais e à busca de alternativas frente ao crescimento populacional e ao esgotamento dos recursos existentes. No meio acadêmico, a sua introdução remonta a Lessig (2008), mediante o argumento de que existem duas economias: a comercial e a compartilhada. A economia comercial é permeada pela lógica do mercado, em que os produtos e serviços possuem valor monetário tangível, seja dinheiro ou trabalho. Já a economia compartilhada identifica as transações que não envolvem trocas monetárias, sendo regulada por um conjunto de relações sociais (amizades e regras de convívio social) e não por métricas de preço.

Os termos mesh economy (Gansky, 2010), consumo colaborativo (Botsman \& Rogers, 2009), consumo conectado (Dubois, Schor, \& Carfagna, 2014) e elancing (Aguinis \& Lawal, 2013) também enfatizam o compartilhamento como elemento central das transações. Portanto, podem ser considerados definições próximas (senão equivalente) ao conceito de economia compartilhada, o qual ganhou maior proeminência devido ao seu nome mais apelativo (Sundararajan, 2016).

A economia compartilhada é com frequência interpretada incorretamente como sinônimo de conceitos correlatos que identificam aspectos específicos relacionados à modificação na natureza das transações econômicas, propiciadas pela utilização das novas tecnologias digitais. Cabe a realização de uma distinção entre esses conceitos a seguir.

1) Economia de plataforma (plataforma digital ou economia de plataforma on-line): se mostra mais amplo e engloba todas as transações on-line comerciais e não comerciais.

2) On-demand economy: remete ao fornecimento digital de atividades que buscam atender à demanda do consumidor por meio do acesso imediato e flexível a bens e serviços (por exemplo: maquiagem, entrega de refeições, reparos manuais).

3) Gig economy: reúne as formas de trabalho mais flexíveis e temporárias que surgiram em resposta ao avanço nas transações on-line (Mulcahy, 2016).

4) Peer-to-peer economy: enfatiza o crescente caráter descentralizado e pontual apresentado pelas transações on-line (Aslam \& Shah, 2017).

A economia compartilhada está diretamente relacionada ao movimento crowd e ao conceito de crowd economy, termo cunhado por Reinaldo Pamponet, em 2011, para identificar o desenvolvimento de uma nova economia, dotada de novos valores de troca que surgem de movimentos coletivos fortemente permeados pela interação entre as pessoas.

O conceito de crowd economy se soma às teorias microeconômicas do valor-trabalho e da utilidade marginal, oferecendo uma resposta à tragédia dos bens comuns, para a qual os bens comuns tendem a ser utilizados de forma excessiva. De acordo com Benkler $(2002,2006)$, a tecnologia de rede consegue evitar o consumo excessivo desses bens por meio da denominada produção de bens comuns por pares em rede.

Diferentemente das formas tradicionais de propriedade, nas quais os bens são excludentes (se é meu, não é seu), na produção por pares em rede eles são inclusivos (é de todos nós). Isto é, qualquer pessoa pode ter acesso aos bens, desde que respeite as regras estabelecidas (Bauwens, 2006). A definição das regras corretas de acesso representa elemento-chave, responsável por promover a utilização eficiente desses bens. A emergência e o sucesso das novas tecnologias digitais possibilitam 
o desenvolvimento de regras mais sofisticadas, o que ajuda a explicar o rápido avanço da economia compartilhada a partir da década de 1990.

Cabe salientar que não existe um consenso na literatura de que o termo economia compartilhada é o mais apropriado. Kaplan (2014) e Hamari, Sjöklint, e Ukkonen (2016), por exemplo, argumentam que esse termo é utilizado de forma inadequada para descrever atividades humanas caracterizadas pelo surgimento de novas formas de organização do trabalho e pelo compartilhamento de capacidade produtiva excedente em troca de dinheiro. O resultado é a emergência de uma economia caracterizada pela realização de transações ponto a ponto (peer-to-peer - p2p), mais horizontalizadas e descentralizadas.

Segundo Bradshaw e Brook (2014) e Eckhardt e Bardhi (2015), as atividades relacionadas à economia compartilhada na realidade se baseiam na disponibilização do acesso a ativos subutilizados, sendo intermediadas pelo mercado a partir de plataformas digitais e empresas especializadas. Assim, o termo economia do acesso se mostra mais adequado.

No tocante à governança pública, a literatura tem demonstrado que a economia compartilhada pode contribuir para a melhoria na gestão dos governos locais (Clarke, Lindquist, \& Roy, 2017; Ganapati \& Reddick, 2018; Hong \& Lee, 2018; Hong \& Ryu, 2019; Selloni, 2017; Tomkinson, 2017). Apesar de existir uma literatura internacional crescente que analisa os efeitos do compartilhamento sobre o setor público, não foi encontrado nenhum estudo na literatura nacional sobre o tema. Assim, este artigo tem como objetivo introduzir a literatura de compartilhamento de ativos públicos em território nacional, buscando conscientizar os gestores públicos sobre os diferentes modos pelos quais a economia compartilhada pode contribuir para a melhoria nos serviços ofertados.

Para isso, o artigo realiza uma pesquisa documental (Marconi \& Lakatos, 2002) por meio do levantamento dos aplicativos privados de compartilhamento que surgiram na última década em âmbito nacional e internacional. Os dados apresentados são obtidos por meio da realização de uma pesquisa em artigos e relatórios técnicos, sendo as informações pertinentes levantadas em jornais, sites e páginas de referência na internet. Os aplicativos são classificados em diferentes categorias, de acordo com as características exibidas.

Ademais, uma regressão por mínimos quadrados ordinários (MQO) é estimada para identificar se a digitalização dos serviços municipais influencia no desempenho fiscal dos municípios brasileiros, mensurado através do Índice FIRJAN de Gestão Fiscal (IFGF). A vantagem apresentada pela regressão é que ela permite calcular o impacto gerado pela digitalização dos serviços públicos sobre o desempenho fiscal dos municípios. Isso justifica a utilização desse ferramental metodológico para identificar o efeito das novas tecnologias digitais sobre a gestão fiscal dos municípios brasileiros. Os dados utilizados foram extraídos da base on-line disponibilizada pelo Instituto Brasileiro de Geografia e Estatística (IBGE) para o ano de 2014, para 5.465 municípios, ano em que o IBGE coletou dados sobre fornecimento de serviços públicos por meio da Pesquisa de Informações Básicas Municipais (MUNIC).

Além desta Introdução, o artigo se encontra estruturado em mais cinco seções. Inicialmente, a seção 2, Economia Compartilhada, apresenta os principais estudos sobre essa forma de economia. Na sequência, a seção 3, Governança Pública e Compartilhamento, mostra o modo como a economia compartilhada está contribuindo para a melhoria na gestão pública local. Posteriormente, a seção 4 , Resultados Encontrados para as Estatísticas Descritivas e para a Regressão Estimada, traz os resultados obtidos para a regressão estimada. Concluindo, a seção 5 expõe as Considerações Finais deste trabalho. 


\section{ECONOMIA COMPARTILHADA}

A aceleração na expansão da economia compartilhada, impulsionada pelos avanços tecnológicos, se dá a partir da década de 1990 nos Estados Unidos. O desenvolvimento das tecnologias de informação, em especial da internet, propiciou o surgimento de um novo padrão de consumo baseado no acesso aos bens e serviços, que torna desnecessária a aquisição dos bens (Schor, 2016).

Grosso modo, a economia compartilhada é caracterizada por duas dimensões-chave. Primeiro: ela depende centralmente da internet para a realização das transações. Segundo: o compartilhamento dos ativos gera valor. Isto é, os ativos são alugados em vez de compartilhados, de modo que as transações são comerciais em vez de gratuitas (Kaplan, 2014; Sundararajan, 2016).

Para Gansky (2010), a economia compartilhada representa uma nova tendência em forte processo de expansão, principalmente nos países desenvolvidos, nos quais emerge na forma de novas organizações e novos modelos de negócios, com foco no compartilhamento de recursos humanos e físicos, incluindo a criação, produção, distribuição, comércio e consumo compartilhado de bens e serviços por pessoas e organizações. Ela também é definida por Dubois et al. (2014) como um conjunto de iniciativas de consumo conectado que promove a reutilização de produtos, elimina intermediários e cria mecanismos de interação ponto a ponto (P2P).

A sua expansão é reforçada pela difusão das tecnologias de comunicação, principalmente dos smartphones e do mercado de aplicativos (Ganapati \& Reddick, 2018). Com a maior utilização dos dispositivos móveis e com a difusão da banda larga sem fio, dos smartphones e dos sensores conectados à internet surge a economia de aplicativos móveis e serviços baseados em localização. Os smartphones possibilitam novas oportunidades para a rede P2P e os sensores de localização e monitoramento capturam as informações de localização dos usuários em tempo real, sendo essas informações utilizadas para o fornecimento de serviços personalizados (Ganapati, 2015).

As empresas de economia compartilhada usam a internet para fornecer as plataformas e gerar as informações que possibilitam o estabelecimento de conexões entre pessoas e empresas ao longo do tempo e do espaço. Por sua vez, as plataformas proporcionam a infraestrutura tecnológica necessária para a promoção do compartilhamento, das interações, da comunicação e da participação em rede. Ela se mostra multifacetada, reunindo diferentes grupos de produtores e consumidores com o objetivo de agrupar os usuários e promover o compartilhamento de bens e serviços (Ganapati, 2015).

As transformações provocadas por essa nova tendência são de tamanha magnitude que Rifkin (2016) chega a argumentar que ela representa a emergência de um novo modelo de produção póscapitalista. Com efeito, a economia compartilhada cresceu exponencialmente na última década, processo que tende a se intensificar com o avanço na inteligência artificial, na indústria 4.0 e nas impressoras 3D. Inclusive, o valor de mercado de algumas das plataformas de economia compartilhada já ultrapassa o valor de firmas estabelecidas no setor. A Uber, por exemplo, surgiu em 2009 e em 2018 valia US\$ 68 bilhões, valor superior ao de cada uma das três maiores empresas estadunidenses de automóveis: Chrysler, Ford e General Motors (Chen, 2015). O Airbnb, que surgiu em 2008, em 2018 estava avaliado em US\$ 30 bilhões, valor mais elevado que o da cadeia de hotéis Hilton e quase o valor da cadeia de hotéis Marriott (Ganapati \& Reddick, 2018).

Para além da utilização de novas tecnologias digitais para desenvolver regras mais eficientes de acesso aos bens e serviços, o avanço da economia compartilhada também é reforçado por três tendências hodiernas: 1) a crescente consciência sobre a necessidade de adoção de hábitos mais sustentáveis (Bradshaw \& Brook, 2014); 2) a mentalidade característica da geração Y, que possui maior facilidade em utilizar 
novas tecnologias (Bull, 2010; Rifkin, 2016); e 3) a crise internacional de 2008, que criou um campo fértil para a utilização de novas tecnologias com o objetivo de racionalizar o consumo (Marshall, 2015).

Além desses, há ainda três fatores adicionais que contribuem para o sucesso dos aplicativos de compartilhamento.

1) A eliminação dos intermediários: a facilidade de comunicação gerada pelos smartphones significa que as pessoas podem comercializar bens diretamente a partir de plataformas digitais especializadas (Rifkin, 2016);

2) A redução na assimetria de informação: possibilitada pelo surgimento de aplicativos que disponibilizam informações em tempo real sobre os usuários (Weber, 2014);

3) O monitoramento e a utilização de métricas de mensuração da reputação: eliminam o comportamento oportunista, ajudam a resolver o problema do caroneiro (Sundararajan, 2016; Thierer, Koopman, Hobson, \& Kuiper, 2015) e possibilitam a oferta privada de bens comuns (Benkler, 2002).

A grande restrição que existia ao avanço dos aplicativos de compartilhamento era a presença de comportamento oportunista, que impossibilita a oferta de bens públicos. A solução encontrada pela economia compartilhada é a construção de métricas de mensuração da reputação dos usuários. A pressão gerada pela avaliação e pela possibilidade de não poder desfrutar dos benefícios propiciados pelos aplicativos de compartilhamento estimula cada usuário a adotar comportamentos que maximizem a avaliação recebida. De modo que a criação de métricas de acesso na forma de mecanismos de mensuração da reputação possibilita a oferta de bens por meio de aplicativos, conferindo segurança e viabilizando a realização das transações (Botsman, 2017; Gandini, 2016).

O valor dos aplicativos de compartilhamento se eleva quando são colocados critérios mais rígidos que impedem que pessoas com comportamento oportunista voltem a utilizá-los. A reputação do aplicativo é dada pela reputação dos usuários, de modo que sua sobrevivência e seu crescimento dependem da adoção de critérios rígidos e transparentes, que elevem a qualidade dos serviços oferecidos e incentivem os prestadores de serviços a maximizar a experiência dos usuários (Botsman, 2017).

Em termos cronológicos, o surgimento da economia compartilhada pode ser discriminado conforme apresentado a seguir. Em meados da década de 1990, o aparecimento da internet estimulou o comércio eletrônico, surgindo os primeiros mercados on-line P2P, como a Amazon e o eBay. Nos anos 2000 nasceram as mídias sociais: LinkedIn, em 2002; Orkut e Facebook, em 2004; Twitter, em 2006, e WhatsApp, em 2009. Essas inovações possibilitaram a comunicação P2P dentro de redes colaborativas pessoais e profissionais (Tapscott \& Williams, 2008).

No início da década de 2000 também surgiram as primeiras plataformas de compartilhamento de mídias e conteúdo digital, entre as quais se destacam: Flickr e Vimeo, criadas em 2004; YouTube, 2005; SlideShare, 2006; Pinterest, 2010; Instagram, 2010; Snapchat, 2011; e Tik Tok, 2016. E as plataformas de elaboração de conteúdo coletivo, como: Blogger, 1999; Wikipédia, 2001; Topcoder, 2001; Google Docs, 2006; GitHub, 2008, e Waze, 2009.

Com o lançamento do iPhone, em 2007, começaram a surgir aplicativos voltados para celulares, fato que viabilizou o rápido desenvolvimento do mercado de aplicativos de compartilhamento e o surgimento de aplicativos responsáveis pelo fornecimento de marketplaces especializados na realização de transações específicas. Como resultado, se observa a rápida diversificação nas iniciativas de compartilhamento, que ocorre na forma de uma onda de surgimento de startups especializadas. O Quadro 1 apresenta as principais startups que surgiram no período 2008-2019, discriminadas de acordo com o ramo de atividade em que atuam. 
RAP | Economia compartilhada e governança pública

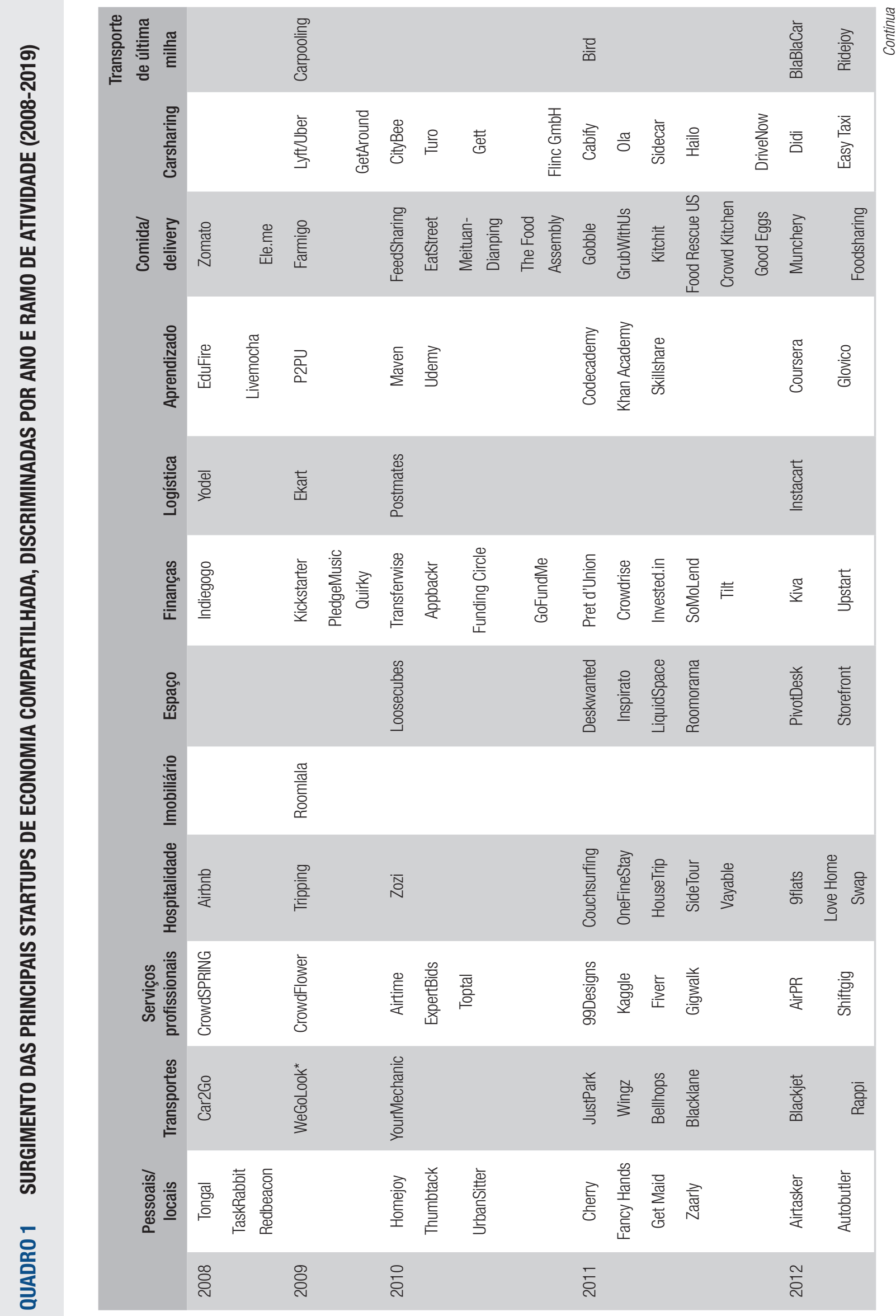

ReVista de AdMinistração PÚBlica | Rio de Janeiro 54(5):1207-1238, set. - out. 2020 
RAP | Economia compartilhada e governança pública

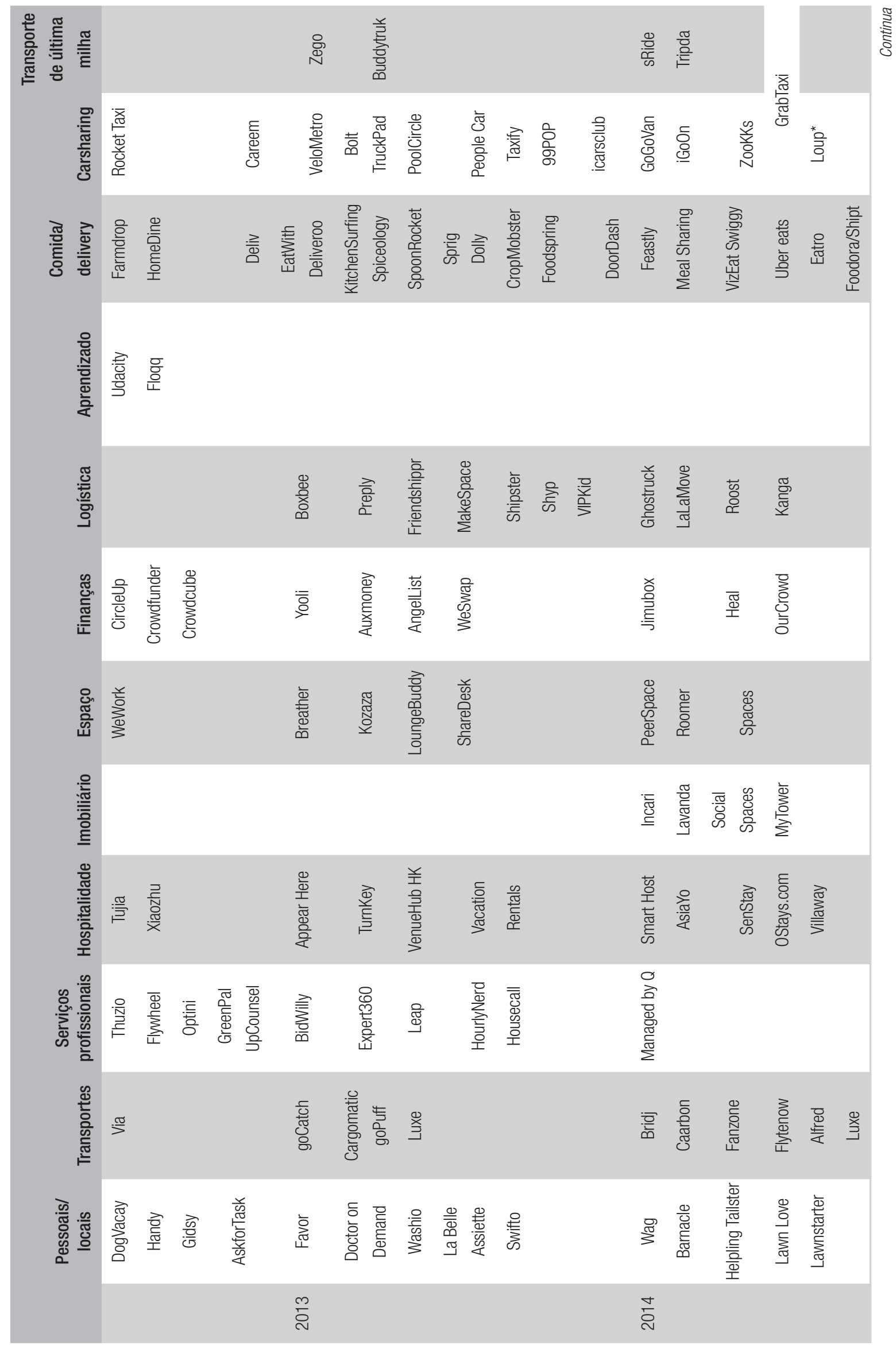

ReVISTA De Administração PÚBlica | Rio de Janeiro 54(5):1207-1238, set. - out. 2020 
RAP | Economia compartilhada e governança pública

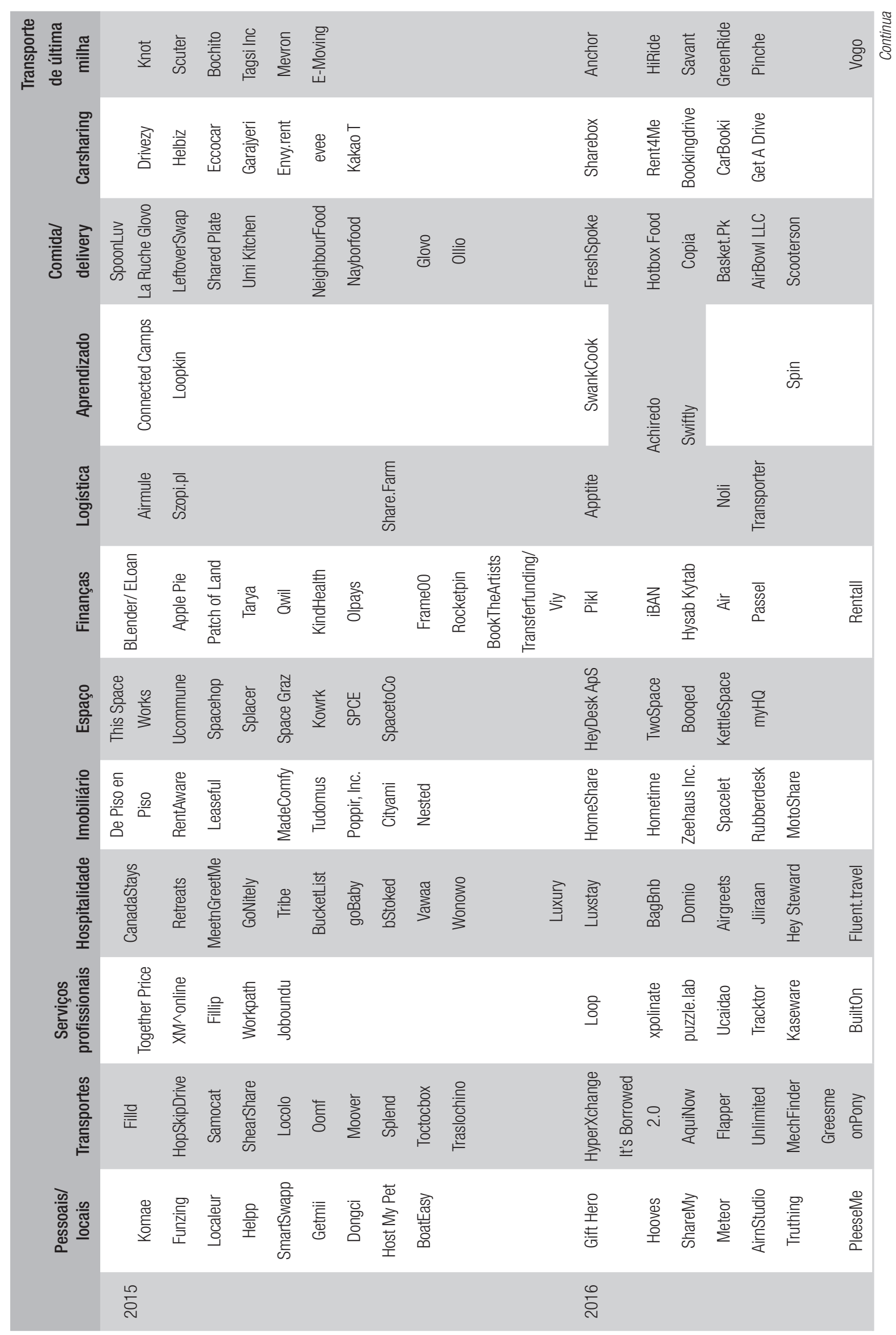

ReVista de Administração Pública | Rio de Janeiro 54(5):1207-1238, set. - out. 2020 
RAP | Economia compartilhada e governança pública

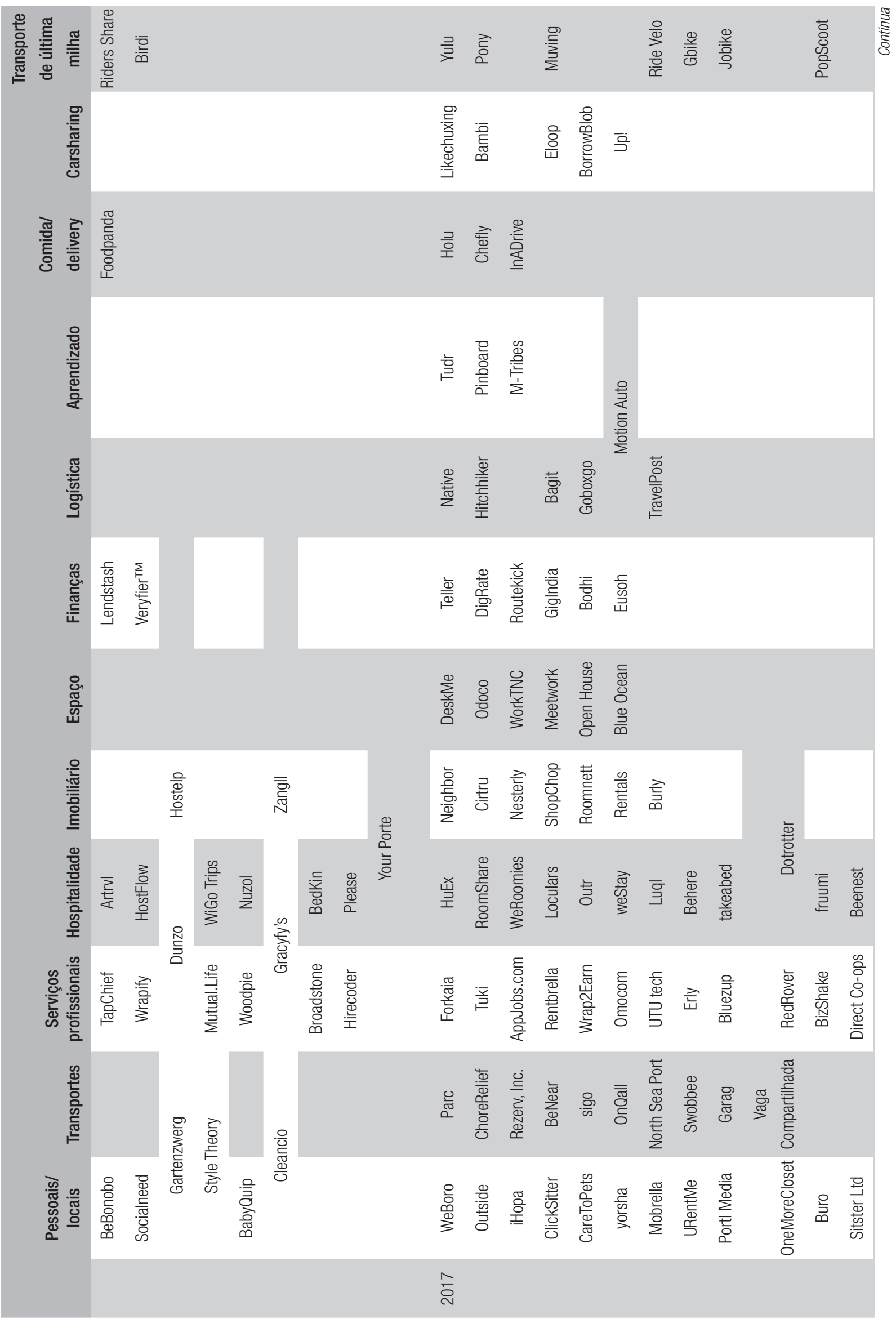

Revista de Administração PÚBlica | Rio de Janeiro 54(5):1207-1238, set. - out. 2020 
RAP | Economia compartilhada e governança pública

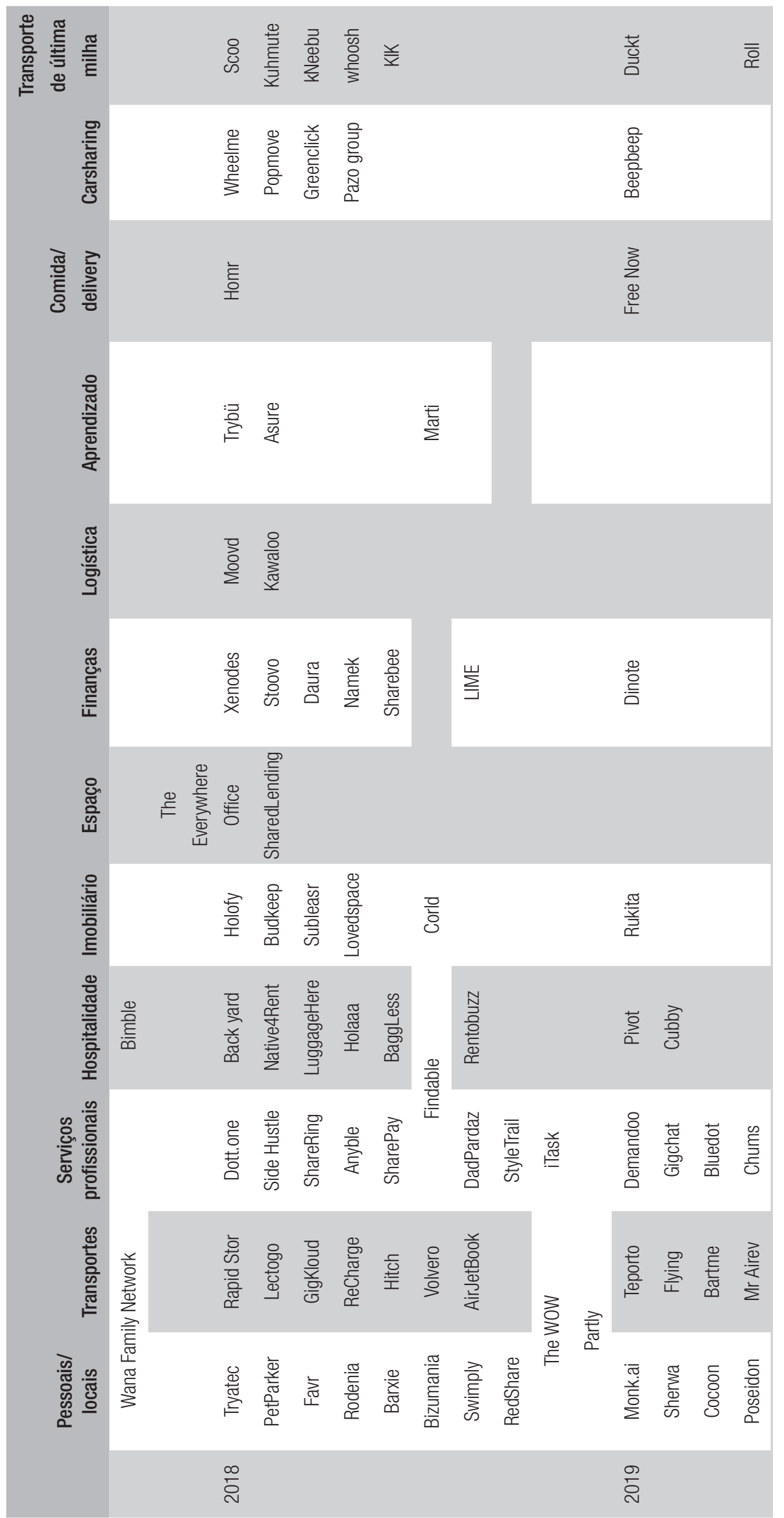


Entre as atividades econômicas nas quais se vislumbra a emergência de startups de economia compartilhada se destacam as plataformas especializadas no compartilhamento de:

- Serviços pessoais/locais: Tongal e TaskRabbit, criadas em 2008; Homejoy, ZOZI, Thumbtack e UrbanSitter, 2010; Cherry, 2011; Airtasker e DogVacay, 2012; Favor e Doctor on Demand, 2013; Lawn Love, 2014; Localeur, 2015; Gift Hero, Hooves, ShareMy, 2016; WeBoro, Outside, iHopa, ClickSitter, 2017; Tryatec, PetParker, Rodenia, Barxie, 2018; Monk.ai, Sherwa, Cocoon, Poseidon, 2019.

- Transporte: Car2Go, 2008; JustPark, Wingz, Bellhops, Blacklane, 2011; Blackjet, Rappi, Via, 2012; goCatch, Cargomatic goPuff, Luxe, 2013; Bridj, Caarbon, Fanzone, Flytenow, 2014; Filld, HopSkipDrive, BoatEasy, ShearShare, 2015; Gartenzwerg, Style Theory, 2016; Parc, ChoreRelief, Rezerv, BeNear, Sigo, 2017; Rapid Stor, Lectogo, GigKloud, ReCharge, Hitch, Volvero, AirJetBook, 2018; Teporto, Flying, Bartme, Mr Airev, 2019.

- Serviços profissionais: crowdSPRING, 2008; CrowdFlower, 2009; Airtime e ExpertBids, 2010; 99Designs e Kaggle, 2011; AirPR, Shiftgig e Thuzio, 2012; BidWilly e HourlyNerd, 2013. Managed by Q, 2014; Together Price, $\mathrm{XM}^{\wedge}$ online, Fillip, Workpath, Joboundu, 2015; Loop, Xpolinate, Puzzle. lab, Ucaidao, Tracktor, 2016; Forkaia, Tuki, AppJobs.com, Rentbrella, 2017; Dott.one, Side Hustle, ShareRing, 2018; Demandoo, Chums, Bluedot, Gigchat, 2019.

- Hospitalidade: Airbnb, 2008; Tripping, 2009; Couchsurfing, OneFineStay, HouseTrip, 2011; 9flats, Love Home Swap, Tujia, Xiaozhu, 2012; Appear Here, TurnKey Vacation Rentals, VenueHub HK, 2013; Smart Host, AsiaYo, SenStay, 2014; CanadaStays, Retreats, MeetnGreetMe, GoNitely, Tribe, 2015; Luxstay, BagBnb, Domio, 2016; HuEx, RoomShare, WeRoomies, Loculars, 2017; Back yard, Native4Rent, LuggageHere, 2018; Pivot, Cubby, 2019.

- Serviços imobiliários: Roomlala, 2009; Incari, Lavanda, Social Spaces, MyTower, 2014; De Piso en Piso, RentAware, MadeComfy, 2015; HomeShare, Hometime, Spacelet, Rubberdesk, MotoShare, 2016; Neighbor, Cirtru, Nesterly, 2017; Holofy, Budkeep, Subleasr, Lovedspace, 2018; Rukita, 2019.

- Espaço: Loosecubes, 2010; Deskwanted, Inspirato, LiquidSpace, Roomorama, 2011; PivotDesk, Storefront, WeWork, 2012; Breather, Kozaza, LoungeBuddy, ShareDesk, 2013; PeerSpace, Roomer, Spaces, 2014; This Space Works, Ucommune, Spacehop, Splacer, 2015; HeyDesk ApS, TwoSpace, myHQ, 2016; DeskMe, Odoco, WorkTNC, Meetwork, Open House, Blue Ocean, 2017; The Everywhere Office, 2018.

- Finanças: Lending Club, 2006; Transferwise, 2010; Pret d’Union, 2011; Kiva, Upstart, em 2012; Yooli e Auxmoney, 2013; Jimubox, 2014; Blender, ELoan, Apple Pie, Patch of Land, Pret d'Union, Tarya e Transferfunding, 2015; Pikl, Lendstash, Veryfier ${ }^{\mathrm{mm}}$, Hysab Kytab, 2016; Teller, Eusoh, DigRate, 2017; Xenodes, Stoovo, Daura, 2018; Dinote, Variable Labs, 2019.

- Logística: Postmates, 2010; Bellhops e Instacart, 2012; Boxbee, Deliv, Friendshippr, MakeSpace, Shipster e Shyp, 2013; Ghostruck, LaLaMove e Roost, 2014; Airmule, Szopi.pl, 2015; Apptite, Achiredo Technologies, Swiftly, Noli, Transporter, 2016; Native, Hitchhiker, Routekick, 2017; Moovd, kawaloo, 2018.

- Aprendizado: EduFire, Livemocha, 2008; P2PU, 2009; Maven e Udemy, 2010; Codecademy, Khan Academy e Skillshare, 2011; Coursera, Glovico e Udacity, 2012; Connected Camps, Loopkin, 2015.

- Comida/delivery: Zomato, Ele.me, 2008; FeedSharing, EatStreet, Meituan-Dianping, 2010; Gobble, GrubWithUs e Kitchit, 2011; Munchery, 2012; EatWith, KitchenSurfing, SpoonRocket e Sprig, 2013; Feastly, Meal Sharing e VizEat, 2014; La Ruche, 2015; FreshSpoke, Hotbox Food, 2016; Basket.Pk, AirBowl LLC, SwankCook, 2017; Holu, Chefly, 2019; Homr, 2019. 
- Carsharing: Lyft, Uber, GetAround, 2008; CityBee, Turo, Gett, Flinc GmbH, 2009; Cabify, Ola, Sidecar, 2010; Didi, Easy Taxi, Rocket Taxi, 2011; VeloMetro, Bolt, Taxify, 99POP, 2013; GoGoVan, iGoOn, ZooKKs, GrabTaxi, Loup, 2014; Drivezy, Eccocar, 2015; Sharebox, Rent4Me, 2016; Likechuxing, Bambi, M-Tribes2017; Wheelme, Popmove, Greenclick, Pazo group, 2018; Beepbeep, Free Now, 2019.

- Transporte de última milha/Caronas: Carpooling, 2009; Bird, 2011; BlaBlaCar, Ridejoy, Deliv, 2012; Zego, 2013; sRide, Tripda, 2014; Knot, Scuter, Bochito, Tagsi, Mevron, E-Moving, 2015; Anchor, HiRide, Savant, GreenRide, 2016; Yulu, Pony, InADrive, 2017; Scoo, Kuhmute, kNeebu, 2018; Duckt, Roll, 2019.

A rápida emergência e disseminação das plataformas de compartilhamento mostra que as transformações geradas pela economia compartilhada são transversais à economia tradicional. Elas abrangem diferentes atividades, culminando em contribuições que se avultam e que possuem impacto crescente sobre a dinâmica econômica.

\section{GOVERNANÇA PÚBLICA E COMPARTILHAMENTO}

De forma semelhante ao setor privado, a economia compartilhada também registra o surgimento de startups com soluções que prometem contribuir para o aumento na eficiência e na qualidade dos bens e serviços públicos. A presente seção revisa essas iniciativas, identificando os desafios e as oportunidades relacionadas ao setor público.

Para compreender o efeito da economia compartilhada sobre o setor público é preciso retomar alguns estudos consagrados na literatura de economia e governança pública. Conforme destacado por Lloyd (1833) e resgatado por Hardin (1968), o livre acesso aos bens comuns tende a levar à superexploração, pois os benefícios advindos desses bens são individuais ao passo que os custos são distribuídos entre todos que os utilizam. De modo que os bens comuns precisam ser privatizados e/ou regulamentados para que a sua exploração ocorra de forma eficiente. Posteriormente, Ostrom, Burger, Field, Norgaard, e Policansky (1999) mostram que os agentes desenvolvem soluções para o problema dos bens comuns. A definição de regras de acesso possibilita a sua utilização de forma coletiva. Ademais, Axelrod (1984) defende que, ao buscar maximizar os ganhos privados, os agentes são incentivados a cooperar, pois o autocontrole coletivo e a cooperação se mostram vantajosos para os indivíduos.

Mais recentemente, Benkler (2002) argumenta que estão surgindo novos modelos de produção de bens comuns, denominados "produção de bens comuns por pares em rede". Nesses sistemas, os bens são organizados de forma descentralizada, colaborativa e não proprietária, sem a necessidade de intervenção do governo. A modularização e a ampla disponibilidade de informações possibilitam a identificação das melhores tarefas pelos agentes. Já o desenvolvimento de mecanismos de acompanhamento das atividades, como a revisão de pares e o controle quantitativo das colaborações, são suficientes para que as transações sejam realizadas de forma eficiente. Ademais, Surowiecki (2007) mostra que são necessárias três condições para que um grupo de pessoas consiga agir coletivamente, tomando decisões eficientes sem a necessidade de supervisão/intermediação de um agente externo: diversidade de opiniões, independência e descentralização.

A economia compartilhada, por meio do uso intensivo da internet e do desenvolvimento de novos sistemas de monitoramento, consegue identificar e classificar os usuários em um sistema de reputação, apresentando mecanismos de autorregulação com propriedades semelhantes às observadas para a 
produção de bens comuns por pares em rede (Botsman, 2017; Sundararajan, 2015). Esses sistemas são utilizados para mitigar o problema do caroneiro e para complementar os governos locais no fornecimento de bens de serviços tipicamente públicos (Weber, 2014).

O avanço da economia compartilhada cria importantes debates sobre o papel a ser desempenhado pelos governos locais, em termos de regulamentação. Tal fenômeno pode ser ilustrado pelo avanço de empresas como a Uber, que ao estimular a realização de transações P2P altamente descentralizadas obriga os governos a revisar seus instrumentos tradicionais de regulamentação (Conselho Administrativo de Defesa Econômica [CADE], 2018; Esteves, 2015; Lei nº 13.640, 2018; Mendes \& Ceroy, 2015; Nota Técnica no 39/2018, 2018; Resende \& Lima, 2018; Resolução nº 148, 2019; Terrien, Maniak, Chen, \& Shaheen, 2016), conferindo maior liberdade para as empresas desenvolverem mecanismos próprios de governança (Bond, 2015; Cohen \& Sundararajan, 2015; Rauch \& Schleicher, 2015).

Apesar da literatura de economia compartilhada ser otimista e defender que a autorregulação reduzirá a necessidade de governos locais (Cannon \& Summers, 2014; Cramer \& Krueger, 2016; Koopman, Mitchell, \& Thierer, 2015; Möhlmann \& Geissinger, 2018; Puschmann \& Alt, 2016; Schor, 2016; Sundararajan, 2017), alguns autores se mostram reticentes. Stemler (2017a, 2017b) e Harris (2017), por exemplo, identificam falhas nos aplicativos de compartilhamento e mostram que os sistemas de reputação e avaliação permanecem sujeitos à manipulação e à presença de vieses cognitivos. Já Zrenner (2015), Smith e Leberstein (2015), Codagnone, Abadie, e Biagi (2016), Erickson e Sørensen (2016) e Harris (2017) mostram que a economia compartilhada cria novos desafios, como a crescente precarização nas relações de trabalho e a necessidade de proteção dos consumidores e dos direitos de propriedade.

Dellarocas (2010) e Brescia (2016) defendem que esse caráter dual da economia compartilhada, caracterizado pela presença simultânea de benefícios e novos desafios, demanda o desenvolvimento de mecanismos de regulamentação governamental mais flexíveis e inteligentes. Os gestores públicos precisam permanecer abertos e inovadores, adotando regulações que promovam a melhoria nos sistemas de reputação, a experimentação e a inovação institucional. No entanto, eles também devem identificar as necessidades pontuais de intervenção e regulamentação, conciliando a adoção de medidas que facilitem o avanço da economia compartilhada com aquelas que busquem corrigir as falhas e anomalias decorrentes.

A literatura especializada também mostra que as soluções propostas pelas empresas de economia compartilhada podem contribuir para o avanço nos instrumentos de gestão pública municipal (Clarke et al., 2017; Hong \& Lee, 2018; Hong \& Ryu, 2019; Selloni, 2017; Tomkinson, 2017; Zon, 2015). Segundo Moon et al. (2014), o advento da internet, em meados da década de 1990, expandiu o escopo do governo digital para o governo eletrônico, com foco em serviços externos aos cidadãos. A partir de meados dos anos 2000, com o surgimento dos novos aplicativos de compartilhamento, o governo passou a ser visto como uma plataforma para a coprodução interativa com os cidadãos, utilizando as tecnologias de informação para solucionar problemas públicos (Albinsson \& Perera, 2012; Heinrichs, 2013; Kirchherr, Reike, \& Hekkert, 2017; Linders, 2012; Zeemering \& Delabbio, 2013).

Atualmente, eles contam com muitas possibilidades de criação de plataformas de compartilhamento para fins específicos, a depender da necessidade e das especificidades dos serviços públicos ofertados aos cidadãos. De acordo com Chasin e Scholta (2015) e Turban, Outland, King, Lee, Liang, e Turban (2018), essas plataformas podem ser classificadas de acordo com o modo como promovem a interação entre os entes públicos e privados. Entre as diferentes formas possíveis de interação, as mais relevantes são o compartilhamento do governo com o governo (government to government - G2G), do governo 
com os cidadãos (government to citizens - G2C), do governo com as empresas (government to business - G2B) e entre os próprios cidadãos (citizens to citizens - C2C).

Entre as iniciativas de compartilhamento à disposição do setor público se destacam: 1) o uso de plataformas de compartilhamento de carros de terceiros, que podem reduzir drasticamente o tamanho das frotas dos governos locais; e 2) o uso de serviços como Lyft, Zipcar e Uber a fim de reduzir o custo de gerenciamento de uma frota própria, cortando despesas de capital e despesas operacionais (Accenture, 2016).

Por exemplo, na área da baía de São Francisco os funcionários dos governos locais estão utilizando o Lyft para ofertar transporte público, reduzir custos e facilitar o deslocamento dos funcionários públicos. De modo semelhante, a cidade de Chicago utiliza o Zipcar para atender a algumas das necessidades de sua frota, o que resulta em economia de US $\$ 0,25$ por milha em relação à propriedade e à operação de sua própria frota (Accenture, 2016).

Outra possibilidade é o aluguel de veículos entre agências e/ou municípios - G2G. Na última década, surgiram diversas plataformas especializadas no compartilhamento de bens e serviços públicos, entre as quais se destacam a ShareOhio.gov e a MuniRent. O objetivo desses aplicativos é difundir o compartilhamento de equipamentos e ferramentas entre os governos locais (Accenture, 2016; Clark et al., 2017; Wosskow, 2014). Também existem aplicativos especializados no compartilhamento de equipamentos médicos G2B, como as plataformas digitais Cohealo e Kaiser Permanente.

Já o compartilhamento G2G de locais de trabalho, por meio de aplicativos como o LiquidSpace, e o aluguel de espaços privados, por meio do Airbnb, resulta na utilização mais eficiente dos escritórios e reduz o número de imóveis necessários. As ferramentas baseadas em nuvem, os aplicativos móveis e o acesso sem fio em escritórios permitem que os funcionários trabalhem em qualquer lugar, reduzindo os custos com espaços físicos (Accenture, 2016; Coleman, 2013; Ganapati \& Reddick, 2018).

A utilização da internet das coisas e de blockchains e a integração e abertura de bases de dados do governo também podem propiciar o desenvolvimento de aplicativos responsáveis por promover a interação direta entre o governo e os cidadãos - G2C (Cherry \& Pidgeon, 2018; Janssen, Konopnicki, Snowdon, \& Ojo, 2017). Entre as vantagens geradas por essas formas de compartilhamento se destacam: 1) a maior qualidade, transparência e eficiência na prestação dos serviços públicos; 2) a simplificação de processos legais e de elaboração de contratos; 3) a segurança; 4) a maior participação da população na elaboração e na aprovação de leis; 5) a redução na poluição e nos engarrafamentos; 6) o maior rastreamento de surtos de doenças; 7) os ganhos de eficiência energética; 8) o rastreamento das transações financeiras; e 9) o desenvolvimento de novos serviços intensivos em informação (Allessie, Sobolewski, \& Vaccari, 2019; Carter, 2018; Hou, 2017; Jun, 2018; Lyons, Courcelas, \& Timsit, 2018; Ojo \& Adebayo, 2017; Ølnes, Ubacht, \& Janssen, 2017; Rivera, Robledo, Larios, \& Ávalos, 2017).

Entre os aplicativos de blockchain utilizados pelos governos locais se destacam:

- Propy, para a emissão de títulos e contratos instantâneos;

- Burstiq, Essentia Framework e MedRec, para compartilhamento de informações médicas;

- Voatz e Follow My Vote, para processos eleitorais mais seguros e flexíveis;

- Waltonchain, monitoramento na emissão de resíduos;

- Crypto Valley, registro de identidade;

- Essentia, controle de fronteira;

- Hyperledger Fabric, rastreamento de créditos de carbono;

- Blockchain Food Safety Alliance, segurança alimentar; 
- Gov2Go e VoiceMap HK, acesso a serviços públicos; e

- Louis Dreyfus Co., gerenciamento de commodities agrícolas.

Outro campo relacionado à gestão pública local que observa elevado crescimento é o compartilhamento entre os cidadãos (consumers to consumers ou citizens to citizens - C2C) de bens tipicamente públicos, por meio da redução ou da redefinição no papel desempenhado pelos governos locais. Esse fenômeno é facilitado pelo maior acesso à internet, crescente facilidade de compartilhamento de informações, desenvolvimento de sensores e de mecanismos digitais de monitoramento e da capacidade de autorregulação das plataformas digitais (Sundararajan, 2016).

Nesse sentido, entre as iniciativas existentes se destacam os aplicativos de compartilhamento de wi-fi (Open Garden e Fon); dados (OpenDataSoft); empréstimos destinados à produção de energia solar (Mosaic); e compartilhamento local de energia (Gridmates, Trec e Vandebron). Esses aplicativos, ao se utilizarem da "sabedoria das multidões" (Surowiecki, 2006; Tapscott \& Williams, 2008) para descentralizar a tomada de decisões, contribuem para a maior eficiência nos governos locais.

Também se observa um movimento incipiente de utilização dos aplicativos para gerar uma massa crescente de dados que pode ser empregada pelo governo para monitorar os serviços públicos, reduzindo a necessidade de geração, armazenamento e gerenciamento de dados próprios (Accenture, 2016). Os dados fornecidos pelos cidadãos a partir de plataformas como a SeeClickFix e a NextDoor podem ajudar a orientar a realização de serviços públicos e o planejamento de longo prazo. Também possibilitam o maior envolvimento da população nas decisões públicas (Accenture, 2016).

Embora o governo não possa depender totalmente das informações fornecidas pelos aplicativos de compartilhamento, a utilização dos dados gerados a partir dessas plataformas pode exercer impacto positivo e significativo na forma como os serviços são projetados e entregues. Além de reduzir os custos com infraestrutura, a exploração das possibilidades criadas pelos ecossistemas digitais propicia o maior envolvimento dos cidadãos nas decisões públicas, fazendo com que as informações e decisões sejam tomadas de forma cada vez mais descentralizada. Os dispositivos móveis criam a possibilidade de os cidadãos se tornarem cocriadores de serviços e políticas públicas (Accenture, 2016).

Além dos aplicativos citados, existem diversos outros que buscam promover a integração social, facilitando o acesso dos cidadãos aos gestores e às informações geradas pelo setor público. $\mathrm{O}$ DriveBC, por exemplo, é utilizado pelo governo da Colúmbia Britânica, no Canadá, para facilitar o planejamento de rotas, através do fornecimento de informações sobre as vias em tempo real. O CitySourced e o Snapp, Send Solve são ferramentas de relatórios para ajudar as pessoas a denunciar questões cívicas, incluindo segurança pública e questões ambientais.

$\mathrm{Na}$ Irlanda, o Noise App é utilizado para relatos de barulho muito alto. O FixMyStreet e o Street Bump permitem às pessoas reportar problemas encontrados nas ruas. O OpenPlans é uma ferramenta de planejamento e transporte. O Outbarriers fornece autonomia para deficientes visuais. O Electore.Me é utilizado no debate de assuntos políticos e sociais. O NationBuilder reúne um grupo de ferramentas de código aberto para comunidades, incluindo mapas, pesquisas e atualizações que facilitam a interação entre agências e indivíduos. O OpenPublic possibilita interação direta com o público. O Open311 é um sistema de relatórios e rastreamento para questões cívicas.

Aplicativos com diferentes ferramentas, como o Oxford City Council, o Kony e o Bradford Metropolitan District Council, auxiliam seus usuários em diversas tarefas, desde a denúncia de pichações até registro de leitores. Já aplicativos como o Open City reúnem voluntários para o desenvolvimento de aplicativos de acordo com os pedidos recebidos dos governos locais. 
Nesse tocante, uma área com elevado potencial de crescimento é a abertura de bases de dados públicos para que desenvolvedores privados criem aplicativos que ampliem as funcionalidades e as formas pelas quais os dados são utilizados. O desenvolvimento de parcerias público-privadas especializadas na gestão de bases de dados e no fornecimento de informações pode contribuir para a maior aplicação dos dados públicos e para o desenvolvimento de soluções e serviços inovadores. Entre as iniciativas pioneiras identificadas se destacam: a cidade de Nova York, que abriu os dados de seu sistema de metrô, e a Cidade-Estado de Singapura, que abriu os dados de seu sistema de táxis.

Com efeito, os aplicativos de compartilhamento abarcam uma multitude de iniciativas que aproximam a população dos governantes. Os cidadãos e as empresas passam a participar ativamente na identificação e no desenvolvimento de soluções para os problemas públicos e na coprodução dos serviços governamentais (Chasin \& Scholta, 2015). O desenvolvimento de aplicativos de compartilhamento governo a governo $(\mathrm{G} 2 \mathrm{G})$ também pode aproximar os diferentes entes que compõem cada governo local, reduzindo a subutilização de ativos e a sobreposição de funções e atribuições.

Conforme destacado por Janssen e Joha (2006) e Meilă (2018), o compartilhamento de serviços representa um grande desafio em termos de governança pública. Diferentes entes de um mesmo governo apresentam estruturas e regras internas específicas, possuindo recursos, objetivos e capacidades que diferem entre si. A utilização de serviços compartilhados requer uma estrutura de governança que seja capaz de resolver diferentes necessidades e requisitos das organizações públicas envolvidas, sendo uma das barreiras que restringem o avanço da economia compartilhada.

O compartilhamento de recursos resulta em novos problemas relativos à maneira como os serviços podem ser compartilhados e coordenados entre diferentes órgãos públicos. Sendo assim, demanda acompanhamento de profissionais com conhecimento especializado e com as habilidades necessárias ao desenvolvimento de soluções para os problemas de coordenação que emergem (Accenture, 2016; Meilă, 2018).

Muitos gestores públicos argumentam que não estão preparados e não possuem os recursos necessários à aplicação dos princípios de economia compartilhada. Em resposta a esses gestores, Accenture (2016) recomenda a adoção do seguinte cronograma para mitigação dos entraves que emergem:

1) Utilizar plataformas como Uber e Lyft, para transporte de primeira e última milha; Airbnb para obter abrigos de emergência após desastres naturais; e MuniRent para compartilhar equipamentos pesados;

2) Quando surgir a necessidade de comprar ativos, primeiro se perguntar se é possível tomar emprestado ou alugar, pois as possibilidades de compartilhamento são quase ilimitadas;

3) Emprestar os ativos que já possui, identificar recursos subutilizados e recorrer ao compartilhamento para obter uma fonte adicional de receitas; e

4) Utilizar aplicativos como NextDoor e SeeClickFix para se conectar aos cidadãos.

\section{RESULTADOS ENCONTRADOS PARA AS ESTATÍSTICAS DESCRITIVAS E PARA A REGRESSÃO ESTIMADA}

Como a economia compartilhada é uma tendência emergente, não existem dados que possibilitem a realização de uma análise mais detalhada da sua contribuição para o avanço nas práticas de gestão adotadas pelos municípios brasileiros. Dada essa limitação, esta seção realiza um levantamento das startups nacionais relacionadas à economia compartilhada, voltadas para a governança local, analisa se os municípios brasileiros fornecem serviços pela internet e estima uma regressão por MQO para identificar o efeito da informatização dos serviços públicos sobre o desempenho fiscal dos municípios. 


\subsection{A economia compartilhada nos municípios brasileiros}

As startups de economia compartilhada voltadas para a governança pública, identificadas a partir do levantamento realizado, se encontram consolidadas a seguir, sendo discriminadas de acordo com a natureza dos serviços fornecidos.

- Fornecimento de informações aos munícipes: Prefeitura de Araras, CCC - Olímpia, Conectaí, CC - Louveira, CCC - Jaguariúna, Oxy Cidadão, Cidadão Mais BRASIL, Olhos de Águia, Minha Cidade, Onde Ir Cidades, Siga Cidades, Alerta Cidadão, ESPIA AQUI, App do Prefeito, ApPREF, Prefeitura mais digital, \#EuFaçoPOA, Boletim ao Mar, Consulta Protocolo, COR.Rio;

- Solicitação de serviços municipais: Vitória Online, Portal Cidadão Campinas, MG App - Cidadão, Curitiba App, SP serviços, Blumenau Cidade Jardim, Prefeitura Na Mão, SP156, 1746 Rio, Prefeitura 24Horas, Jaraguá na Mão, Aracruz Online, Prefeitura de Jundiaí, SEI!, Rio+ Respeito Oficial, Monitora Jampa, USCS Frequência, Aplicativo Escolar, Niterói Rotativo, Detran.SP, HidroWeb;

- Realização de denúncias: Coopera Simões Filho, eOuve, 156 Mobile Campinas, Central 156, Curitiba 156, 156Foz, 156 Sumaré, 156 SJC, Caraguatatuba 156, Fala Feira 156, 156 Limeira, Fiscalize Fortaleza;

- Relato de problemas públicos: Alô Governante, Alô Deputado, Alô Governador, Fala Cidadão, SAAE Agora Itabirito, Fogo Cruzado, OTT, SP+Segura, Colab, Conecta Jacareí, Denúncia Ambiente, App Barueri, Prefeitura de Eusébio, Prefeitura.com.vc, Transalvador, Vitória Online, Bairro Seguro, PBH APP, Pelas Ruas, Alerta Cidadão, Goiás Seguro, Jaguariúna (maus-tratos de animais), Aviso Polícia, CHAMAR 192, Salve Maria, PenhaS, SOS Mulher, Apoio Vítima, BemQuererMulher, MUSA;

- Ferramentas de gestão municipal: Vereador Reconhecido, Servidor.Rio, MarQPonto, Almoxarifado, Colabore, GovFácil, Elotech BI, App Gestão Cidades, Gestão na Mão, Atende.Net, App Pompéu, App Divinópolis, e-BI Inteligência Municipal, APP Cidadão - Franca, Atende.Net, GRP Mobile, SIGRC, Pública Mobile, Escola Virtual Gov, Licit Mais Brasil, Alerta Licitação, EngeGOV, Super Vereador, NeritPolítica, Politique, divoem, Cartão Qualidade, SisDC, Zul+, Gestão Mais BRASIL, Cidadão Mais BRASIL, Veredas Formativa, e-SUS AB Território, ACS - Agente Comunitário de Saúde, ACS Lite eSUS AB ePHealth, ePHealth ACS-Prefeituras, G-MUS Atenção Primária, Saúde Domiciliar;

- Serviços de saúde: BoaConsulta, Busca Medicamentos Campinas, GliCampinas, Meu INSS, Dr.consulta, Doutor Hoje, G-MUS Saúde Cidadão, Agenda Fácil, Hora Marcada, Saúde Já, Doutore, Consultório, Saúde Mental, Saúde Fácil Goiânia, Saúde-Guarulhos, Saúde+ Uberlândia, Saúde Goiás+, Saúde-Valinhos, Saúde Suzano, Net Consulta, Saúde Na Mão SJC, Boraceia Saúde Online, Saúde Digital Ribeirão Preto, Meu digiSUS, Meu Info Saúde, Pronto mobile, Acesso Cidadão, Fala Saúde-Guarapuava, SissOnline Minha Saúde, Saude.rio, São Bernardo Saúde, e-Cidadão Saúde, De olho na consulta, Viconsus, Fast Cidadão;

- Identificação e contratação de serviços médicos de profissionais da saúde e centros médicos próximos: Nossa Saúde, Doctoralia Brasil, Abertta Saúde, Guia de Saúde, Salut Saúde, Saves, Medical, Fácil Consultar, Mediconecta, EmConsulta, Nosso Médico, FastSaúde-ACS, Fast Cidadão, Mediktor, Dokter, Simples Dental, e-Raps, RENAME, ACE-Prefeituras ePHealth, Onde está o dinheiro da saúde?, Cuidar Idoso, Auditor Vetores, mobile AtendSaude Endemias, e-Visit@, evisita, Arbo App, Udi Sem Dengue, Sem Dengue, Patrulha da Dengue, Dengue Não, Valinhos Contra Dengue, Mosquito Zero, Mapa da Dengue, Uberaba Contra a Dengue, Combate Aedes;

- Mobilidade urbana: Giro MetrôRio, Metrô de São Paulo Oficial, Metrô DF, Ônibus.Rio, ÔnibusGV, floripanoponto, PartiuSBC, SiMRmtc, SigaBus Contagem, CittaMobi, Caxias Urbano, Cadê Meu 
Ônibus, Urmob, Wappa, Travessias, SOU, BluMob, Meu Buse, Urmob, Seletivo Fácil Santos, Transporte Coletivo, Meu Destino Vix, Vá de Ônibus, TAXI.RIO, SPTAXI, Taxi 2424, Vá de Táxi, URBS, Taxi62 Faixa Vermelha, Carona Solidária Fiocruz, TopBus+, 4Bus, Busbud, MOTO VIP, Bike Seguro, Bike Itaú, tembici, Riba Share, Grin Scooters, Movo, Lime, Beliive;

- Cidadania e questões cívicas: Chama o Vereador, Meu Vereador, Vereador Em Ação, Vereador Online, Alô Vereador Jandira, eCidadão, Câmara App, Legislador, Infoleg, Câmara Fácil, Fiscaliza Cidadão, Poder do Voto, Câmara Popular, Monitora, Brasil!, Nosso Mandato, Política+, Ação Política, Cidadão Online 4R, Govern, Zap Carioca, 1746 Rio, Governa Santa Rosa, Avise o Prefeito, Prefeitura e Você, EcoRodovias, Participa Campinas, LeisMunicipais, Politize!, Politicometro, Corrida Eleitoral Online, Ranking dos Políticos, Eu Parlamentar, Conheça Seu Candidato, Votodata, Candidato Legal!, Pesquisa Eleitoral App, Teste Político, Minha Cidade, Planet App, SmartCity, Mudamos+;

- Alertas climáticos: AlertaBlu, AlertaRio, AlertaVale, Emergência RJ, Alerta Rio Branco, Mau tempo!, SOS Chuva, Weather Alerts Ultimate, Alerta DCNit, Vai Dar Praia, CEM, ALERTA199, SenseApp, Defesa Civil - Ouro Preto, Alerta DCNit, App Defesa Civil de Aimorés, Sistema de Alertas Rolante-RS; e

- Comunicação de blitz, radares e acidentes: LeisecaMaps, LeiSecaRJ, Blitz Alerta, Blitz Salvador, Detector de Polícia, KoDinMaps, Radardroid, Ray.Radar, Radares, CamSam, Radares Brasil, Glob, AmiGO, Sem Parar.

Essas startups têm como objetivo facilitar o acesso dos cidadãos aos serviços públicos e desenvolver crowd-based solutions. Isto é, utilizar a sabedoria das multidões e as informações descentralizadas, em posse dos cidadãos, para elevar a eficiência na gestão pública. A sua difusão entre os municípios pode contribuir para o avanço na qualidade e na eficiência dos serviços públicos.

Cabe salientar, entretanto, que a comparação com as iniciativas identificadas em âmbito internacional mostra que existem algumas áreas importantes nas quais não foram encontradas iniciativas similares em território nacional - destaque para o aluguel de máquinas e equipamentos entre diferentes entes da federação e para o desenvolvimento de soluções C2C. Por conseguinte, existe espaço para a criação de aplicativos especializados no compartilhamento de ativos públicos entre os municípios brasileiros que estimulem o desenvolvimento de soluções descentralizadas dos cidadãos para os próprios cidadãos.

\subsection{Análise descritiva}

A utilização de novas tecnologias digitais pelos municípios brasileiros é caracterizada por meio da tabulação de algumas estatísticas descritivas, as quais buscam mensurar as características mais pertinentes.

A Tabela 1 mostra que $87 \%$ dos municípios brasileiros possuem serviços de atendimento ao público a distância, por meio da internet. Porém, a disponibilidade desses serviços varia bastante de acordo com o tamanho do município e a região em que se localiza. Os municípios maiores das regiões Centro-Oeste, Norte e Sul são os que se encontram em melhor posicionamento relativo. Observa-se que a proporção dos municípios menores, principalmente das regiões Norte e Nordeste, que fornecem serviços de atendimento ao público a distância, por meio da internet, é inferior ao observado para as demais regiões. Por exemplo, apenas $70 \%$ dos municípios com até 5 mil habitantes da região Nordeste fornecem esse serviço.

REVISTA DE AdMinistração PÚBlicA | Rio de Janeiro 54(5):1207-1238, set. - out. 2020 


\title{
TABELA 1
}

\begin{abstract}
PROPORÇÃO DE MUNICÍPIOS COM SERVIÇOS DE ATENDIMENTO AO PÚBLICO À DISTÂNCIA POR MEIO DA INTERNET, DISCRIMINADO SEGUNDO CLASSES DE TAMANHO DA POPULAÇÃO DOS MUNICÍPIOS (2014)
\end{abstract}

\begin{tabular}{|c|c|c|c|c|c|c|}
\hline & Brasil & Norte & Nordeste & Sudeste & Sul & Centro-0este \\
\hline Total & $87 \%$ & $80 \%$ & $80 \%$ & $92 \%$ & $93 \%$ & $91 \%$ \\
\hline Até 5.000 & $87 \%$ & $77 \%$ & $70 \%$ & $90 \%$ & $93 \%$ & $93 \%$ \\
\hline De 5.001 a 10.000 & $86 \%$ & $73 \%$ & $77 \%$ & $93 \%$ & $91 \%$ & $87 \%$ \\
\hline De 10.001 a 20.000 & $86 \%$ & $78 \%$ & $80 \%$ & $90 \%$ & $95 \%$ & $85 \%$ \\
\hline De 20.001 a 50.000 & $88 \%$ & $82 \%$ & $84 \%$ & $91 \%$ & $94 \%$ & $95 \%$ \\
\hline De 50.001 a 100.000 & $91 \%$ & $86 \%$ & $85 \%$ & $94 \%$ & $96 \%$ & $100 \%$ \\
\hline De 100.001 a 500.000 & $95 \%$ & $100 \%$ & $92 \%$ & $94 \%$ & $94 \%$ & $100 \%$ \\
\hline Mais de 500.000 & $95 \%$ & $100 \%$ & $91 \%$ & $94 \%$ & $100 \%$ & $100 \%$ \\
\hline
\end{tabular}

Fonte: Pesquisa de Informações Básicas Municipais, IBGE.

A análise dos serviços disponibilizados pela internet (Tabelas 2 e 3 ) revela que as novas tecnologias de comunicação ainda são pouco utilizadas pelos municípios para fornecer serviços à população. A grande maioria dos municípios possui páginas na internet, mas os serviços prestados são limitados. Por exemplo, apenas $1 \%$ dos municípios permitem que a matrícula dos alunos e o agendamento de consulta na rede pública de saúde sejam realizados por meio da internet.

A baixa digitalização dos serviços prestados pelos governos locais evidencia a existência de um elevado espaço para a adoção de novas tecnologias digitais e aplicativos de compartilhamento, com vistas a facilitar o acesso da população aos serviços públicos. A adoção de políticas de incentivo ao surgimento de aplicativos privados, especializados no fornecimento de serviços específicos e na interação com a sociedade, pode contribuir para a redução dos custos e para a elevação na qualidade e na eficiência dos serviços públicos prestados. O surgimento de plataformas digitais especializadas pode facilitar o acesso dos cidadãos aos serviços públicos (Accenture, 2016; Cherry \& Pidgeon, 2018). Além disso, também pode propiciar a descentralização e a cocriação desses serviços, por meio da participação ativa da população na identificação, proposição e implementação de políticas públicas voltadas para a solução de problemas de ordem coletiva.

Dada a limitação existente no acesso à internet, o IFGF é utilizado para mensurar as práticas de gestão municipal. Esse índice é elaborado pela Federação das Indústrias do Estado do Rio de Janeiro (FIRJAN) a partir dos resultados fiscais informados pelas próprias prefeituras, disponibilizados anualmente pela Secretaria do Tesouro Nacional (STN). O IFGF é composto por quatro indicadores: Autonomia, Gastos com Pessoal, Liquidez e Investimentos. Cada indicador possui peso de 25\% no índice final.

A grande vantagem apresentada pelo IFGF é a possibilidade de comparação dos municípios, pois se trata de um indicador simples que abrange as diferentes dimensões que caracterizam as finanças públicas municipais, podendo ser utilizado para verificar o modo como a digitalização de serviços públicos afeta o desempenho fiscal. O IFGF varia entre 0 e 1 , sendo classificado em quatro categorias: Conceito A (gestão de excelência), IFGF superior à 0,8 ; Conceito $\mathrm{B}$ (gestão boa), IFGF entre 0,6 e 0,8 ; Conceito C (gestão em dificuldade), IFGF entre 0,4 e 0,6; Conceito D (gestão crítica), IFGF entre 0 e 0,4. Desse modo, é possível comparar diretamente a situação fiscal dos municípios. 
RAP | Economia compartilhada e governança pública

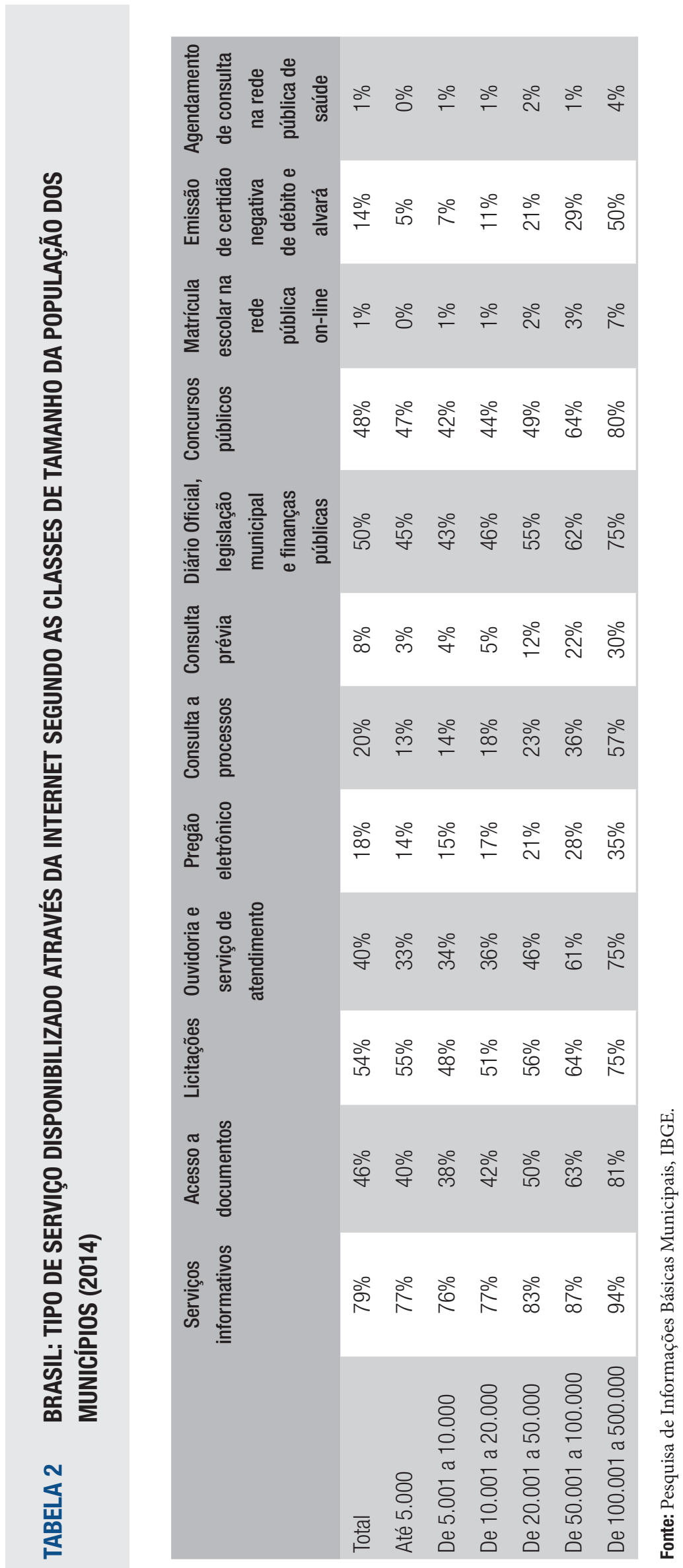

REVISTA DE AdMINIStRação PÚBlica | Rio de Janeiro 54(5):1207-1238, set. - out. 2020 
RAP | Economia compartilhada e governança pública

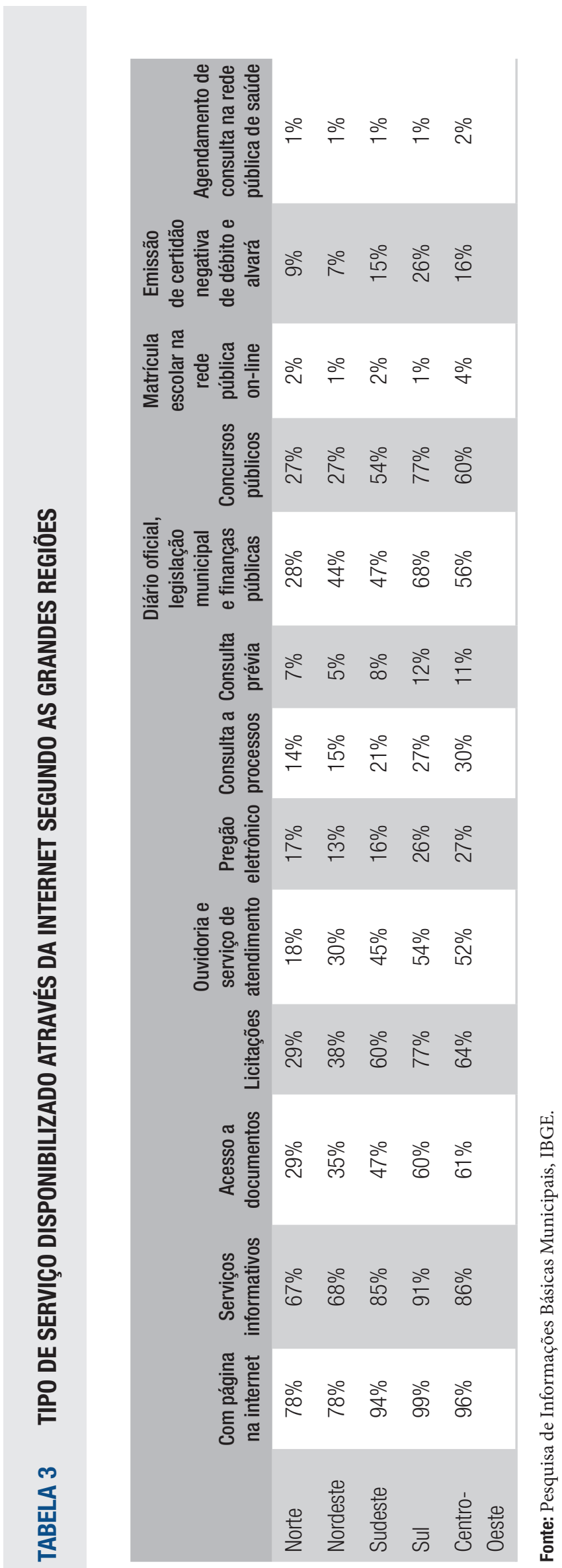

ReVISTA DE AdMINIStração PÚBlicA | Rio de Janeiro 54(5):1207-1238, set. - out. 2020 
A Tabela 4 sintetiza o IFGF dos municípios brasileiros. A partir de 2009, a maioria dos municípios passou a ser classificada com conceito gestão boa, o que é explicado principalmente pela redução dos municípios com gestão em dificuldade e gestão crítica. $\mathrm{O}$ número de municípios com gestão de excelência permanece relativamente baixo, não se observando tendência de elevação na quantidade de municípios classificados nessa categoria.

\section{TABELA 4 EVOLUÇÃO DO IFGF (2005-2016)}

\begin{tabular}{|r|r|r|r|r|r|r|r|r|r|r|r|r|} 
& 2005 & 2006 & 2007 & 2008 & 2009 & 2010 & 2011 & 2012 & 2013 & 2014 & 2015 & 2016 \\
\hline Conceito A & 142 & 198 & 198 & 260 & 316 & 435 & 503 & 568 & 654 & 556 & 296 & 431 \\
\hline Conceito B & 2.105 & 2.282 & 2.637 & 2.618 & 2.961 & 3.002 & 3.147 & 3.296 & 3.413 & 3.521 & 3.615 & 3.741 \\
\hline Conceito C & 2.214 & 2.238 & 2.311 & 2.345 & 2.084 & 1.972 & 1.801 & 1.612 & 1.433 & 1.430 & 1.583 & 1.289 \\
\hline Conceito D & 825 & 615 & 351 & 261 & 125 & 100 & 57 & 36 & 17 & 24 & 27 & 10 \\
\hline
\end{tabular}

Fonte: Adaptado de FIRJAN.

\subsection{Regressão estimada}

Segundo Gujarati (2011), a análise de regressão identifica se existe uma relação de dependência de uma variável, a variável dependente, em relação a uma ou mais variáveis, as variáveis exploratórias, podendo ser formalmente expressa através da equação

$$
Y=X \beta+u
$$

com $Y$ sendo a variável dependente, no presente caso o IFGF; $X$ um vetor de variáveis exploratórias; $\beta$ é o parâmetro estimado; e $u$ é o termo de erro.

$\mathrm{O}$ vetor $\mathrm{X}$ é composto por um conjunto de oito variáveis que identificam o grau de informatização das gestões municipais (correio; fax; jornais; internet; telefone; telefone exclusivo; computadores em rede; intranet); quatorze variáveis referentes ao fornecimento de informações e à digitalização de serviços fornecidos pelos governos locais (página ativa; serviços informativos e notícias; acesso a documentos e formulários; licitações; ouvidoria; pregão eletrônico; consulta a processos; consulta prévia ao alvará provisório; Diário Oficial, legislação municipal e finanças públicas; concursos públicos; matrícula escolar na rede pública on-line; certidão negativa de débito; agendamento de consulta na rede pública de saúde; política de inclusão digital); e três variáveis de controle (logaritmo da população; proporção de CLT; proporção de estatutários), adicionadas para identificar o efeito do tamanho do município e do grau de profissionalização da gestão municipal sobre o desempenho fiscal.

O modelo de regressão é estimado pelo método de MQO, com dados disponibilizados pelo IBGE para 2014, para 5.465 municípios, a partir de dados oriundos da MUNIC. O modelo possui como 
objetivo avaliar o impacto das novas tecnologias digitais sobre a gestão fiscal dos governos locais. $\mathrm{O}$ modelo de regressão é a ferramenta escolhida, pois permite constatar o efeito da digitalização de cada serviço municipal sobre o desempenho fiscal, fornecendo informações elucidativas sobre os ganhos que podem ser obtidos a partir da adoção de uma política de incentivo à utilização de aplicativos de compartilhamento nos governos locais.

O efeito da digitalização de cada serviço sobre o desempenho fiscal é identificado pela aplicação do teste de hipóteses, a saber:

$$
\text { Teste } t=\frac{\hat{\beta}-\beta_{i}}{e p(\beta)} \text {, }
$$

sendo $\hat{\beta}$ o valor encontrado para o parâmetro estimado e ep $(\beta)$ o seu respectivo erro-padrão.

Como a hipótese nula testada é de que o coeficiente $\hat{\beta}$ é igual a zero, a equação (2) colapsa na seguinte equação:

$$
\text { Teste } t=\frac{\hat{\beta}}{e p(\beta)} \text {, }
$$

se o valor encontrado para o Teste $t$ for superior ao tabelado (para distribuição normal) com $n-k$ graus de liberdade, sendo $n$ o número de municípios e $k$ o número de variáveis exploratórias, a hipótese alternativa é corroborada. Caso contrário, não é possível rejeitar a hipótese nula. Ademais, se o valor encontrado para o Teste $t$ for superior ao tabelado e o coeficiente estimado apresentar sinal negativo, há indícios de que a digitalização do serviço compromete o desempenho fiscal do município.

Conforme recomendado por Gujarati (2011), o coeficiente de determinação, o Teste F, o fator de inflação da variância e o teste de Breusch-Pagan são aplicados para identificar se o modelo estimado pode ser utilizado para a realização de inferências estatísticas.

A Tabela 5 apresenta os resultados obtidos para a regressão estimada. No que tange ao fornecimento de serviços pelos governos locais via internet, apenas as variáveis binárias que identificam a presença de fax e telefone não são estatisticamente significativas. Todas as demais variáveis contribuem para a melhoria no desempenho fiscal dos municípios, apesar da variável política de inclusão digital apresentar valor próximo de zero. A maior presença de jornais, internet, intranet, telefone exclusivo, computadores ligados em rede e de página ativa contribui para o melhor desempenho fiscal, apesar de os coeficientes estimados serem próximos a zero. Esses resultados evidenciam os efeitos positivos gerados pela informatização dos governos locais, em termos de melhoria na gestão fiscal dos municípios. 


\section{TABELA 5 REGRESSÃO ESTIMADA PARA O FORNECIMENTO DE SERVIÇOS PELOS GOVERNOS LOCAIS ATRAVÉS DA INTERNET (2014)}

\begin{tabular}{|c|c|c|c|c|c|}
\hline Variável & Coef.\# & 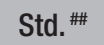 & Variável & Coef. & Std. \\
\hline Correio & $0,018^{* *}$ & 0,009 & Pregão eletrônico & $-0,014^{\star \star}$ & 0,003 \\
\hline Fax & 0,000 & 0,003 & Consulta a processos & $0,008^{\star \star}$ & 0,003 \\
\hline Jornais & $0,021^{\star *}$ & 0,003 & $\begin{array}{l}\text { Consulta prévia (alvará } \\
\text { provisório) }\end{array}$ & $0,008^{\star}$ & 0,005 \\
\hline Internet & $0,023^{* *}$ & 0,003 & $\begin{array}{l}\text { Diário oficial, legislação } \\
\text { municipal e finanças públicas }\end{array}$ & $-0,009^{* \star}$ & 0,003 \\
\hline Telefone & 0,000 & 0,004 & Concursos públicos & $0,031^{\star *}$ & 0,003 \\
\hline Telefone exclusivo & $0,008^{*}$ & 0,004 & $\begin{array}{l}\text { Matrícula escolar na rede } \\
\text { pública on-line }\end{array}$ & 0,004 & 0,010 \\
\hline Computadores em rede & $0,007^{\star *}$ & 0,002 & Certidão negativa de débito & $0,046^{\star \star}$ & 0,004 \\
\hline Intranet & $0,016^{\star *}$ & 0,003 & $\begin{array}{l}\text { Agendamento de consulta na } \\
\text { rede pública de saúde }\end{array}$ & $-0,03^{\star *}$ & 0,013 \\
\hline Página ativa & $0,028^{* \star}$ & 0,004 & Política de inclusão digital & 0,000 & 0,003 \\
\hline Serviços informativos e notícias & $0,016^{\star *}$ & 0,004 & Log população & $-0,002$ & 0,001 \\
\hline Acesso a documentos e Formulários & 0,002 & 0,003 & Proporção de CLT & $0,095^{\star \star}$ & 0,008 \\
\hline Licitações & $0,011^{* *}$ & 0,003 & Proporção de estatutários & $0,146^{\star *}$ & 0,008 \\
\hline Ouvidoria & $0,019^{* *}$ & 0,003 & Constante & $0,487^{\star \star}$ & 0,014 \\
\hline R2 & 0,3273 & & & & \\
\hline Teste F & 105,87 & & & & \\
\hline Breusch-Pagan & 16,25 & & & & \\
\hline
\end{tabular}

Notas: ${ }^{*}$ Significativo ao nível de confiança de $95 \%$.

*Significativo ao nível de confiança de $90 \%$. O fator de inflação da variância evidencia a ausência de multicolinearidade.

"Coeficiente estimado.

\#Erro-padrão.

Fonte: Elaborada pelos autores.

A regressão estimada também verifica se o fornecimento de serviços por meio da página digital dos governos locais contribui para a melhoria no desempenho fiscal. Com exceção do acesso a documentos e formulários e da matrícula escolar na rede pública on-line, todas as demais variáveis binárias são significativas. Porém, pregão eletrônico, Diário Oficial, legislação municipal e finanças públicas e agendamento de consulta na rede pública de saúde apresentam sinal negativo, indicando que o fornecimento desses serviços a partir dos sites resulta em um pior desempenho fiscal. Ademais, política de inclusão digital, consulta a processos e consulta prévia (alvará provisório) exibem coeficientes estimados próximos azero. 
Os resultados negativos encontrados para essas variáveis evidenciam os desafios impostos pelas novas tecnologias digitais à gestão municipal. As características distintas apresentadas pelos entes que compõem cada governo local significam que o compartilhamento e a informatização de serviços podem gerar diversos problemas de gestão (Accenture, 2016). Esses serviços estão relacionados, por exemplo, à necessidade de padronização de rotinas e procedimentos, ao treinamento dos funcionários públicos e à eliminação de problemas de coordenação. Torna-se necessário o devido acompanhamento dos gestores no desenvolvimento de soluções relacionadas ao fornecimento desses serviços.

Os coeficientes negativos são verificados principalmente para aqueles serviços que demandam maior interação com os cidadãos. Por outro lado, os sinais positivos são observados sobretudo para os serviços que demandam menor interação com os cidadãos. De modo que os maiores problemas de gestão local, relacionados à utilização de novas tecnologias digitais, são observados nos serviços que envolvem maior interação com os usuários. Essa é uma evidência favorável ao argumento de que atualmente os municípios não estão preparados para fornecer serviços públicos por meio de ferramentas digitais. Também pode indicar que a criação de ferramentas que aumentam a acessibilidade aos serviços públicos resulta em maior interação com os usuários, o que demanda maior dispêndio de tempo com o atendimento aos usuários, elevando os custos e contribuindo para a deterioração na situação fiscal dos municípios.

Cabe salientar que o desenvolvimento de novas ferramentas que facilitam a interação com os usuários é uma das principais áreas de expansão da economia compartilhada. Os mecanismos de avaliação e autorregulação presentes nessas plataformas podem ajudar a reduzir os problemas de coordenação e interação com os usuários enfrentados atualmente pelos gestores municipais brasileiros (Botsman, 2017; Möhlmann \& Geissinger, 2018; Sundararajan, 2016).

Em relação às variáveis de controle, o $\log$ da população não é significativo, indicando que os problemas de gestão não estão relacionados ao tamanho do município. Por outro lado, as variáveis proporção de trabalhadores CLT e proporção de trabalhadores estatutários são estatisticamente significativas, evidenciando a necessidade de se controlar o efeito da qualificação dos funcionários sobre o desempenho fiscal dos municípios.

Em suma, os resultados encontrados mostram que os municípios enfrentam diversos problemas relacionados à digitalização dos serviços públicos. Os argumentos aqui apresentados corroboram a literatura de economia compartilhada: a adoção de aplicativos de compartilhamento e de instrumentos que possibilitam a interação direta entre os cidadãos pode exercer efeito positivo sobre o fornecimento de serviços públicos, mostrando-se benéfica para os municípios (Accenture, 2016; Chasin \& Scholta, 2015; Ganapati \& Reddick, 2018; Kirchherr et al., 2017; Meilă, 2018; Turban et al., 2018). Justifica-se, assim, o fato dessas áreas se encontrarem entre as que observam maior surgimento de aplicativos de economia compartilhadas voltados para gestão pública em âmbito nacional. De certa forma, o surgimento desses aplicativos já é uma resposta dos gestores públicos a esses problemas. 


\section{CONSIDERAÇÕES FINAIS}

A economia compartilhada identifica um conjunto de atividades que não se restringem, necessariamente, à promoção do compartilhamento de recursos, possuindo como denominador comum a viabilização do acesso a bens e serviços por meio de novas tecnologias digitais. Essas tecnologias são utilizadas para criar mercados virtualizados, constituídos por regras específicas de interação on-line que buscam oferecer uma resposta factível à tragédia dos bens comuns e à presença de agentes oportunistas, possibilitando a oferta de bens públicos por meio do desenvolvimento de aplicativos e plataformas digitais especializadas que incentivam a interação descentralizada e direta entre os cidadãos (C2C), os cidadãos e o governo (C2G), diferentes entes e esferas do governo (G2G) e entre o governo e empresas privadas (G2B).

O presente artigo utiliza a técnica de pesquisa documental para realizar um levantamento dos aplicativos de compartilhamento voltados para o setor público que surgiram recentemente em âmbito nacional e internacional. A análise descritiva de dados e a estimação de uma regressão são utilizadas para caracterizar o acesso à internet em território nacional e para identificar o efeito da digitalização dos serviços públicos sobre o desempenho fiscal dos municípios brasileiros.

O levantamento dos aplicativos de economia compartilhada, realizado na seção 2, mostrou que a partir da década de 1990, com o avanço nas tecnologias de comunicação (internet, smartphones e aplicativos), começaram a surgir startups de compartilhamento especializadas em facilitar o acesso a bens e serviços. Dessa forma, a economia compartilhada se tornou cada vez mais transversal e relevante. O seu avanço é, provavelmente, uma das principais fontes de inovações das duas primeiras décadas do século XXI.

Os dados apresentados na seção 3 mostram que o avanço da economia compartilhada cria importantes constrangimentos e oportunidades para os governos locais. Ela demanda a modificação nas atribuições e cria novos desafios para os governos locais. Também propicia: 1) a coordenação mais eficiente entre as esferas e entes que compõem os governos locais $(\mathrm{G} 2 \mathrm{G}) ; 2$ ) a maior descentralização e acesso dos cidadãos às decisões públicas $(\mathrm{G} 2 \mathrm{C}) ; 3$ ) o desenvolvimento de soluções descentralizadas (C2C) para problemas de ordem pública, reduzindo a necessidade de intermediação do governo; e 4) o surgimento de aplicativos G2B, especializados no fornecimento de soluções que prometem elevar a eficiência do setor público, mediante a redução na subutilização de ativos e coordenação mais eficiente intra e entre entes federativos.

Já a subseção 4.1 mostra que a utilização de aplicativos de economia compartilhada é uma realidade em muitos municípios brasileiros. O levantamento realizado evidenciou o elevado grau de diversificação nas contribuições da economia compartilhada para a melhoria na gestão dos municípios brasileiros. Essas contribuições vão desde aplicativos especializados no fornecimento de informações e agendamento de serviços até aplicativos que estimulam a participação cidadã e a identificação e proposição de soluções para problemas públicos.

A análise realizada na subseção 4.1 também indicou que ainda existe espaço para o surgimento de aplicativos de compartilhamento em atividades como: 1) coordenação entre governos locais; 2) digitalização dos governos locais (conforme evidenciado pelos dados compilados na subseção 4.2);

3) redução na subutilização de ativos públicos; e 4) desenvolvimento de soluções descentralizadas (C2C).

A regressão estimada para verificar o modo como a digitalização dos serviços públicos influencia no desempenho fiscal dos municípios, mensurado a partir do IFGF (subseção 4.3), mostra que o 
fornecimento de serviços de atendimento ao público por meio da internet contribui significativamente para a melhoria nos indicadores de gestão fiscal. Paradoxalmente, a informatização dos serviços públicos, principalmente daqueles que demandam maior interação com os usuários, exerce efeito negativo sobre o desempenho fiscal dos municípios. Esse resultado é favorável ao argumento de que os gestores públicos não estão sabendo explorar as vantagens apresentadas pelas tecnologias digitais, sucumbindo diante dos novos desafios. Eles carecem de iniciativas, instrumentos e políticas públicas que os auxiliem na modernização dos serviços, rotinas e procedimentos realizados, espaço que pode ser ocupado pelos novos aplicativos de compartilhamento.

Com efeito, as evidências encontradas ao longo do artigo mostram que a economia compartilhada está transformando a forma como os governos gerenciam a infraestrutura existente e como ela é utilizada. Também indicam que existe espaço para o desenvolvimento de soluções virtualizadas e para o surgimento de aplicativos especializados no fornecimento de bens públicos. Os aplicativos de economia compartilhada permitem a interação mais descentralizada e a autorregulação dos usuários, podendo ajudar os gestores públicos a enfrentar as dificuldades de gestão e coordenação presentes atualmente. O desenvolvimento de novas regras de acesso a bens públicos encapsuladas em aplicativos e plataformas digitais especializadas representa campo fértil de pesquisa, com elevado potencial de crescimento e que pode contribuir significativamente para o avanço do setor público.

Os gestores públicos municipais também carecem de políticas responsáveis por promover a maior conscientização sobre: 1) os aplicativos privados existentes atualmente; 2) os ganhos que podem ser obtidos com a utilização desses aplicativos; e 3) as possibilidades de adoção de iniciativas de compartilhamento nos governos locais. Também se defende a necessidade de políticas públicas que estimulem o desenvolvimento de plataformas de compartilhamento nos governos locais, principalmente de políticas de fomento ao empreendedorismo, voltadas para o desenvolvimento de aplicativos especializados no compartilhamento, cocriação e descentralização dos ativos e serviços públicos, e que facilitem a realização de parcerias entre as iniciativas pública e privada.

Fica como sugestão para trabalhos futuros a realização de estudos que busquem avançar em temáticas como: identificação de novas possibilidades de utilização da economia compartilhada no setor público; levantamento de dificuldades e desafios enfrentados na utilização de aplicativos de compartilhamento no setor públicos; e proposição de soluções, adaptações e modificações institucionais que potencializem a contribuição da economia compartilhada para a gestão local. 


\section{REFERÊNCIAS}

Accenture. (2016). Is ownership obsolete? Share your way to government innovation. Recuperado de https:// www.accenture.com/t20160224t161639z__w__/ us-en/_acnmedia/accenture/next-gen/insightsharing-economy/documents/accenture-sharingeconomy-letter.pdf

Aguinis, H., \& Lawal, S. O. (2013). eLancing: a review and research agenda for bridging the science-practice gap. Human Resource Management Review, 23(1), 6-17.

Albinsson, P. A., \& Perera, B. Y. (2012). Alternative marketplaces in the 21 st century: Building community through sharing events. Journal of consumer Behaviour, 11(4), 303-315.

Allessie, D., Sobolewski, M., \& Vaccari, L. (2019). Blockchain for digital government: An assessment of pioneering implementations in public services. (JCR Science for policy report). Seville, Spain: European Commission, Joint Research Centre.

Aslam, A., \& Shah, M. A. (2017). Taxation and the peer-to-peer economy. Washington, DC: International Monetary Fund.

Axelrod, Robert (1984). The evolution of cooperation. New York, NY: Basic Books.

Bauwens, M. (2006). The political economy of peer production. post-autistic economics review, 37, 33-44.

Benkler, Y. (2002). Coase's penguin, or, Linux and the nature of the firm. The Yale Law Journal, 112(3), 369-446.

Benkler, Y. (2006). The wealth of networks: how social production transforms markets and freedom. London, UK: Yale University Press.

Bond, A. T. (2015). An app for that: local governments and the rise of the sharing economy. Notre Dame L. Rev. Online, 90(2), 77-96.

Botsman, R. (2017). Who can you trust? How technology brought us together and why it might drive us apart. London, UK: Hachette.

Botsman, R., \& Rogers, R. (2009). O que é meu é seu: como o consumo colaborativo vai mudar o nosso mundo. Porto Alegre, RS: Bookman.

Bradshaw, C. J., \& Brook, B. W. (2014). Human population reduction is not a quick fix for environmental problems. Proceedings of the National Academy of Sciences, 111(46), 16610-16615.

Brescia, R. H. (2016). Regulating the sharing economy: New and old insights into an oversight regime for the peer-to-peer economy. Neb. L. Rev., 95(1), 87-145.

Bull, G. (2010). The always-connected generation. Learning \& Leading with Technology, 38(3), 28-29.

Cannon, S., \& Summers, L. H. (2014). How Uber and the sharing economy can win over regulators. Harvard Business Review, 13(10), 24-28.

Carter, L., \& Ubacht, J. (2018). Blockchain applications in government. In Proceedings of the 19th Annual International Conference on Digital Government Research: governance in the data age, New York, NY, USA.

Chasin, F., \& Scholta, H. (2015). Taking peer-topeer sharing and collaborative consumption onto the next level-new opportunities and challenges for e-government. In Proceedings of the 23rd European Conference on Information Systems (ECIS), Münster, Germany.

Chen, L. (2015, 04 de dezembro). At \$68 billion valuation, Uber will be bigger than GM, Ford, and Honda. Forbes.

Cherry, C. E., \& Pidgeon, N. F. (2018). Is sharing the solution? Exploring public acceptability of the sharing economy. Journal of cleaner production, 195, 939-948.

Clark, B. Y., Larco, N., \& Mann, R. F. (2017, agosto). The impacts of autonomous vehicles and e-commerce on local government budgeting and finance. Portland, Orgen: University of Oregon. Recuperado de https:// ssrn.com/abstract $=3009840$

Clarke, A.; Lindquist, E. A.; Roy, J. (2017). Understanding governance in the digital era: an agenda for public administration research in Canada. Canadian Public Administration, 60(4), 457-476.

Codagnone, C., Abadie, F., \& Biagi, F. (2016). The future of work in the 'sharing economy'. Market efficiency and equitable opportunities or unfair precarisation? (JRC Science for Policy Report). Seville, Spain: Institute for Prospective Technological Studies. 
Cohen, M., \& Sundararajan, A. (2015). Selfregulation and innovation in the peer-to-peer sharing economy. U. Chi. L. Rev. Dialogue, 82(1), 116-133.

Coleman, C. (2013). Total workplace transformsfederal office space. Recuperado de https://gsablogs.gsa. gov/innovation/2013/11/19/gsas-total-workplacecreates-a-21st-centuryworkplace-designed-to-savemoney-and-increase-efficiency-and-productivity/

Conselho Administrativo de Defesa Econômica. (2018). Processo Administrativo $n$. 08700.006964/2015-71. Brasília, DF: Autor.

Cramer, J., \& Krueger, A. B. (2016). Disruptive change in the taxi business: the case of Uber. American Economic Review, 106(5), 177-82.

Dellarocas, C. (2010). Designing reputation systems for the social web. (Research Paper Series, No. 201018). Boston, Massachusetts: Boston University School of Management.

Dubois, E., Schor, J., \& Carfagna, L. (2014, abril). Connected consumption: a sharing economy takes hold. Rotman Management, 1, 50-55.

Eckhardt, G. M., \& Bardhi, F. (2015, 28 de janeiro). The sharing economy isn't about sharing at all. Harvard business review.

Erickson, K., \& Sørensen, I. (2016). Regulating the sharing economy: introduction to the special issue. Internet Policy Review, 5(2), 1-15.

Esteves, L. A. (2015). Rivalidade após entrada: o impacto imediato do aplicativo Uber sobre as corridas de táxi porta-a-porta (Documento de trabalho, 003/2015). Brasília, DF: Conselho Administrativo de Defesa Econômica.

Federação das Indústrias do Estado de Rio de Janeiro. (2017). IFGF 2017: Índice FIRJAN de Gestão Fiscal. Rio de Janeiro, RJ: Autor. Recuperado de: https://www.firjan.com.br/ifgf/downloads/

Frenken, K., \& Schor, J. (2017, junho). Putting the sharing economy into perspective. Environmental Innovation and Societal Transitions, 23, 3-10.

Ganapati, S. (2015). Using mobile apps in government. Washington, DC: IBM Center for The Business of Government.

Ganapati, S., \& Reddick, C. G. (2018). Prospects and challenges of sharing economy for the public sector. Government Information Quarterly, 35(1), 77-87.

Gansky, L. (2010). The mesh: why the future of business is sharing. New York, NY: Penguin Random House.

Gujarati, D. N.; Porter, D. C. (2012). Econometria Básica. (5a Ed.). São Paulo, SP: AMGH Editora.

Hamari, J., Sjöklint, M., \& Ukkonen, A. (2016). The sharing economy: why people participate in collaborative consumption. Journal of the association for information science and technology, 67(9), 20472059.

Hardin, G. (1968). The tragedy of the commons. Science, 162(3859), 1243-1248.

Harris, B. (2017). Uber, lyft, and regulating the sharing economy. Seattle UL Rev., 41(1), 269-285.

Heinrichs, H. (2013). Sharing economy: a potential new pathway to sustainability. GAIA-Ecological Perspectives for Science and Society, 22(4), 228-232.

Hong, S.; Lee, S. (2018). Adaptive governance and decentralization: evidence from regulation of the sharing economy in multi-level governance. Government Information Quarterly, 35(2), 299-305.

Hong, S.; Ryu, J. (2019). Crowdfunding public projects: Collaborative governance for achieving citizen co-funding of public goods. Government Information Quarterly, 36(1), 145-153.

Hou, H. (2017). The application of blockchain technology in E-government in China. In Proceedings of the 26th International Conference on Computer Communication and Networks (pp. 1-4), Honolulu, USA.

Janssen, M., \& Joha, A. (2006, dezembro). Governance of shared services in public administration. In Proceedings of the Americas Conference on Information Systems 2006, (pp. 2306-2314), Acapulco, Mexico.

Janssen, M., Konopnicki, D., Snowdon, J. L., \& Ojo, A. (2017). Driving public sector innovation using big and open linked data (BOLD). Information systems frontiers, 19(2), 189-195.

Jun, M. S. (2018). Blockchain government-a next form of infrastructure for the twenty-first century. Journal of Open Innovation: Technology, Market, and Complexity, 4(7), 1-12. 
Kaplan, D. (2014, 30 de janeiro). Collaboratif Trois questions à... Daniel Kaplan, Fing. Alliancy. Recuperado de https://www.alliancy.fr/article/ industrie/2014/01/30/collaboratif-trois-questionsa-daniel-kaplan-fing

Kirchherr, J., Reike, D., \& Hekkert, M. (2017, dezembro). Conceptualizing the circular economy: an analysis of 114 definitions. Resources, Conservation and Recycling, 127, 221-232.

Koopman, C., Mitchell, M. D., \& Thierer, A. D. (2015, maio). The sharing economy and consumer protection regulations: the case for policy change. The Journal of Business, Entrepreneurship \& Law, 8(2), 529-545.

Lei no 13.640, de 26 de março de 2018. (2018). Altera a Lei $\mathrm{n}^{\circ} 12.587$, de 3 de janeiro de 2012, para regulamentar o transporte remunerado privado individual de passageiros. Brasília, DF: Diário Oficial da União.

Lessig, L. (2008). Remix: making art and commerce thrive in the hybrid economy. New York, NY: The Penguin Press.

Linders, D. (2012). From e-government to wegovernment: defining a typology for citizen coproduction in the age of social media. Government Information Quarterly, 29(4), 446-454.

Lloyd, W. F. (1833). Two lectures on the checks to population. Oxford, UK: Oxford University Press.

Lyons, T., Courcelas, L., \& Timsit, K. (2018, 07 de dezembro). Blockchain for government and public services (Thematic Report). Paris, France: The European Union Blockchain Observatory \& Forum.

Marconi, M. D. A., \& Lakatos, E. M. (2002). Técnicas de pesquisa (Vol. 2, pp. 35-36). São Paulo, SP: Atlas.

Marshall, P. (2015, 03 de agosto). The sharing economy: is it really different from traditional business? SAGE Business Researcher. Recuperado de http://businessresearcher.sagepub.com/sbr-164596738-2690068/20150803/the-sharing-economy\#

Meilă, A. D. (2018). Sustainable urban mobility in the sharing economy: digital platforms, collaborative governance, and innovative transportation. Contemporary Readings in Law and Social Justice, 10(1), 130-136.

Mendes, F. S., \& Ceroy, F. M. (2015). Economia compartilhada e a politica nacional de mobilidade urbana: uma proposta de marco legal (Texto para Discussão $\mathrm{n}^{\circ}$ 185). Brasília, DF: Núcleo de Estudos e Pesquisas/CONLEG/Senado.

Möhlmann, M., \& Geissinger, A. (2018). Trust in the sharing economy: platform-mediated peer trust. Cambridge Handbook of the Law of the Sharing Economy. Cambridge, MA: Cambridge University Press.

Moon, M. J., Lee, J., \& Roh, C.-Y. (2014). The evolution of internal IT applications and e-government studies in public administration: Research themes and methods. Administration \& Society, 46(1), 3-36.

Mulcahy, D. (2016). The gig economy: the complete guide to getting better work, taking more time off, and financing the life you want. New York, NY: Amacom.

Nota Técnica no 39/2018/DEE/CADE. (2018). Ato de concentração referente à operação de transferência de autorização para prestação de serviços de transporte interestadual de passageiros da Gardênia para a UTIL. Análise de diferença de médias de linhas, passageiros e descontos dos períodos pré e pós-operação por mínimos quadrados ordinários. Não se encontrou, de forma consistente, qualquer mudança de padrão de atuação que possa ter decorrido da referida operação. Brasília, DF: Conselho Administrativo de Defesa Econômica.

Ojo, A.; Adebayo, S. (2017). Blockchain as a next generation government information infrastructure: A review of initiatives in D5 countries. In A. Ojo, \& J. Millard (Eds.), Government 3.0-Next Generation Government Technology Infrastructure and Services (pp. 283-298). Cham, Germany: Springer.

Ølnes, S.; Ubacht, J.; Janssen, M. (2017). Blockchain in government: Benefits and implications of distributed ledger technology for information sharing. Amsterdam, The Netherlands: Elsevier.

Ostrom, E., Burger, J., Field, C. B., Norgaard, R. B., \& Policansky, D. (1999). Revisiting the commons: local lessons, global challenges. Science, 284(5412), 278-282.

Puschmann, T. \& Alt, R. (2016). Sharing economy. Business \& Information Systems Engineering, 58(1), 93-99.

Rauch, D., \& Schleicher, D. (2015). Like Uber, but for local governmental policy: the future of local regulation of the 'sharing economy'. (Research Paper, 15-01). Fairfax, Virginia: George Mason University. 
Resende, G. M., \& Lima, R. C. A. (2018). Efeitos concorrenciais da economia do compartilhamento no Brasil: a entrada da Uber afetou o mercado de aplicativos de táxi entre 2014 e 2016? (Documento de trabalho, n. 001/2018). Brasília, DF: Conselho Administrativo de Defesa Econômica.

Resolução no 148, de 2 de agosto de 2019. (2019). Altera a Resolução CGSN no 140, de 22 de maio de 2018, que dispõe sobre o Regime Especial Unificado de Arrecadação de Tributos e Contribuições devidos pelas Microempresas e Empresas de Pequeno Porte (Simples Nacional). Brasília, DF: Diário Oficial da União. Recuperado em http://www.in.gov. br/web/dou/-/resolucao-n-148-de-2-de-agostode-2019-209517410

Rifkin, J. (2016). Sociedade com custo marginal zero. São Paulo, SP: M. Books do Brasil Editora.

Rivera, R., Robledo, J. L., Larios, V. M., \& Ávalos, J.M. (2017). How digital identity on blockchain can contribute in a smart city environment. In Proceedings of 2017 International Smart Cities Conference. (pp. 1-4), Wuxi, China.

Schor, J. (2016, outubro). Debating the sharing economy. Journal of Self-Governance \& Management Economics, 4(3), s.p.

Schor, J. B., Fitzmaurice, C., Carfagna, L. B., Attwood-Charles, W., \& Poteat, E. D. (2016). Paradoxes of openness and distinction in the sharing economy. Poetics, 54, 66-81.

Selloni, D. (2017). New forms of economies: sharing economy, collaborative consumption, peer-to-peer economy. In D. Selloni. (Ed.), CoDesign for PublicInterest Services (pp. 15-26). Cham, Germany: Springer.

Smith, R., \& Leberstein, S. (2015). Rights on demand: Ensuring workplace standards and worker security in the on-demand economy. Washington, DC: National Employment Law Project.

Stemler, A. (2017a). Feedback loop failure: Implications for the self-regulation of the sharing economy. Minn. JL Sci. \& Tech., 18(2), 673-712.

Stemler, A. (2017b). The myth of the sharing economy and its implications for regulating innovation. Emory Law Journal, 67(2), 197-241.
Sundararajan, A. (2016). The sharing economy: The end of employment and the rise of crowd-based capitalism. Cambridge, MA: Mit Press.

Sundararajan, A. (2017, fevereiro). The collaborative economy: socioeconomic, regulatory and policy issues. Bruxelas, Belgic: Directorate General for Internal Policies, Policy Department A: Economic and Scientific Policy, European Parliament.

Surowiecki, J. (2006). A sabedoria das multidões. Rio de Janeiro, RJ: Record.

Tapscott, D., \& Williams, A. D. (2008). Wikinomics: How mass collaboration changes everything. New York, NY: Penguin Random House.

Terrien, C., Maniak, R., Chen, B., \& Shaheen, S. (2016, setembro). Good practices for local governments and private companies driving change together in urban mobility: lessons learned from one-way carsharing. California, USA: University of California.

Thierer, A., Koopman, C., Hobson, A., \& Kuiper, C. (2015). How the internet, the sharing economy, and reputational feedback mechanisms solve the lemons problem. U. Miami L. Rev., 70(3), 830-878.

Tomkinson, R. (2017). Shared services in local government: improving service delivery. London, UK: Routledge.

Tribunal de Contas da União (2015). Política pública de inclusão digital. Brasília, DF: Autor.

Turban, E., Outland, J., King, D., Lee, J. K., Liang, T. P., \& Turban, D. C. (2018). Innovative EC Systems: From E-Government to E-Learning, E-Health, Sharing Economy, and P2P Commerce. In E. Turban, J. Outland, D. King, J. K. Lee, T. P. Liang, \& D. C. Turban. (Eds.), Electronic Commerce 2018 (pp. 167201). Cham, Germany: Springer.

Weber, T. A. (2014). Intermediation in a sharing economy: insurance, moral hazard, and rent extraction. Journal of Management Information Systems, 31(3), 35-71.

Wosskow, D. (2014). Unlocking the sharing economy: an independent review. London, UK: Department for Business, Innovation and Skills.

Zeemering, E. S., \& Delabbio, D. (2013). A county manager's guide to shared services in local government. Washington, DC: IBM Center for the Business of Government. 
RAP | Economia compartilhada e governança pública

Zon, N. (2015, dezembro). The sharing economy and why it matters for policy makers. London, UK: Public Sector Digest.
Zrenner, A. (2015). The ethics of regulating the Sharing Economy. Durham, North Carolina: The Kenan Institute for Ethics at Duke University.

\section{Adilson Giovanini}

https://orcid.org/0000-0001-8948-1186

Professor colaborador da Universidade do Estado de Santa Catarina (UDESC); Doutorado em economia pelo Programa de Pós-graduação em Economia da Universidade Federal de Santa Catarina (UDESC).

E-mail: adilsoneconomia@gmail.com 